\title{
An Ensemble Rate Adaptation Framework for Dynamic Adaptive Streaming over HTTP
}

\author{
Hui Yuan, Senior Member, IEEE, Xiaoqian Hu, Junhui Hou, Member, IEEE, Xuekai Wei, \\ and Sam Kwong, Fellow, IEEE
}

\begin{abstract}
Rate adaptation is one of the most important issues in dynamic adaptive streaming over HTTP (DASH). Due to the frequent fluctuations of the network bandwidth and complex variations of video content, it is difficult to deal with the varying network conditions and video content perfectly by using a single rate adaptation method. In this paper, we propose an ensemble rate adaptation framework for DASH, which aims to leverage the advantages of multiple methods involved in the framework to improve the quality of experience $(Q o E)$ of users. The proposed framework is simple yet very effective. Specifically, the proposed framework is composed of two modules, i.e. the method pool and method controller. In the method pool, several rate adaptation methods are integrated. At each decision time, only the method that can achieve the best $Q o E$ is chosen to determine the bitrate of the requested video segment. Besides, we also propose two strategies for switching methods, i.e., InstAnt Method Switching, and InterMittent Method Switching, for the method controller to determine which method can provide the best $Q o E s$. Simulation results demonstrate that, the proposed framework always achieves the highest $Q o E$ for the change of channel environment and video complexity, compared with stateof-the-art rate adaptation methods.
\end{abstract}

Index Terms-Dynamic adaptive Streaming over HTTP (DASH), Quality of experience $(Q o E)$, Rate adaptation, Video compression, and Video transmission.

\section{INTRODUCTION}

$\mathbf{I}$ $\mathrm{N}$ the last few years, video streaming has become the dominant source of traffic over the Internet. To face this growing demand of media traffic, new efficient video streaming techniques have been developed, such as the popular Dynamic Adaptive Streaming over HTTP (DASH) [1]. In contrast to RTP/UDP, DASH is easy to configure and, in particular, can greatly simplify the traversal of firewalls and network address translators. In addition, it can be easily deployed with content

Manuscript Received

This work was supported in part by the National Key R\&D Program of China under Grants 2018YFC0831003, in part by the National Natural Science Foundation of China under Grants 61571274 and 61871342; in part by the Shandong Natural Science Funds for Distinguished Young Scholar under Grant JQ201614; in part by the Young Scholars Program of Shandong University (YSPSDU) under Grant 2015WLJH39.

H. Yuan is with the School of Control Science and Engineering, Shandong University, Ji'nan 250061, China (Email: huiyuan@sdu.edu.cn).

$\mathrm{X}$. Hu is with the School of Information Science and Engineering, Shandong University, Qingdao, 266237, China (Email: huxiaoqian11@163.com).

J. Hou (corresponding author), X. Wei and S. Kwong are with the Department of Computer Science, City University of Hong Kong, Kowloon, Hong Kong (Email: jh.hou@cityu.edu.hk, xuekaiwei2-c@my.cityu.edu.hk, and cssamk@cityu.edu.hk).

Copyright (c) 2019 IEEE. Personal use of this material is permitted. However, permission to use this material for any other purposes must be obtained from the IEEE by sending an email to pubspermissions@ieee.org.

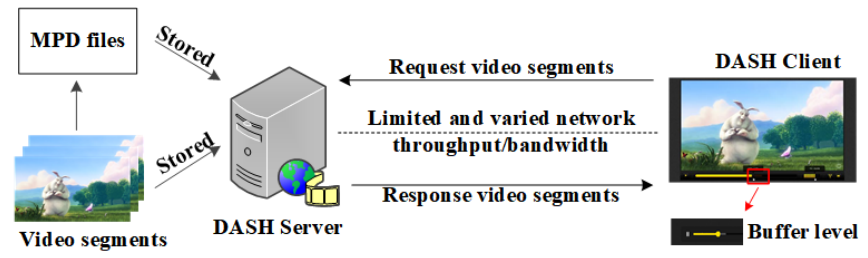

Fig. 1. Structure of a DASH based video delivery system.

delivery networks at a relatively low cost. Therefore, DASH has been widely adopted for providing uninterrupted video streaming services for users [2].

The key concept of DASH is that each video is encoded into several representations with different bitrates. These representations are then divided into small segments or chunks (typically with display time of 1-10 seconds). As shown in Fig. 1, at the beginning of a DASH connection, a manifest file (MPD file) that records the information of all the available chunks of a video, e.g., URL addresses, chunk lengths, quality levels, resolutions, etc., is first downloaded by the client. Then the client will download the MPD file and dynamically request video segments with various bitrates based on its rate adaptation logic. It is noteworthy that the rate adaptation logic of clients is not specified in the DASH protocol. Naive DASH adaptation logics usually suffer from large quality variations or playback buffer underflow [3], which drastically deteriorates the quality of experience $(Q o E)$ of users [4]. Because the network bandwidth is highly dynamic, it is challenging to provide stable and high quality videos to ensure satisfactory user experience all the time. Therefore, how to design appropriate rate adaptation methods to improve user $Q o E$ is one of the most important research topics (see Section II for details) for DASH.

Different from the traditional quality of service $(Q o S)$-based video streaming like RTP/UDP, DASH is designed as a $Q o E$ compatible streaming protocol which can satisfy the greedy requirements of users by using appropriate rate adaptation methods. Arising from the characteristics of human visual system [5], $Q o E$ is affected by lots of factors, e.g., the initial playback delay, the received video quality, smoothness, the number and duration of playback stalling events, the instantaneous interactions between playback stalling, video quality [6-12].

Due to the complexity of network variations, it is difficult to utilize a single method to adapt to the network variations perfectly for all time. Therefore, to achieve the optimal $Q o E$ 
for users as much as possible, we propose an ensemble rate adaptation framework for DASH. Our contributions are as follows.

1) We propose an ensemble rate adaptation framework for DASH to adapt complex network variations in practice, such that the optimal QoE for users can be achieved.

2) We propose two control strategies to select the best method at different decision times to adapt to diverse network conditions.

3) To fit the proposed framework, we also propose a QoE model by taking the average video quality, the quality variation, re-buffering events, and the initial playout delay into account.

The rest of the paper is organized as follows. Related work is discussed in Section II. The proposed framework is presented in detail in Section III. Simulation results and conclusions are given in Sections IV and V, respectively.

\section{RELATED WORK}

Existing rate adaptation methods for DASH can be roughly divided into two categories, i.e., content-dependent methods (usually for multi-view videos and 360-degree videos)[13$16]$ and content-independent methods [6][17-34]. The contentindependent methods can be further classified into two categories, i.e. methods for a single server [6][17-35] and methods for multi-servers [18][36][37]. Since only content-independent methods with a single server are investigated in this paper, only those related works are discussed.

Dräler and Karl [3] introduced the quality-first-based method. In this method, users will always request the video segment whose bitrate best matches the estimated channel bandwidth. If there is not enough channel capacity, it tries to download segments with the next lowest video quality level, until there is no capacity or buffer space left. This algorithm favors downloading segments at higher video quality levels at the expense of having more buffering segments. On the contrary, Huang et al. [19][20] proposed a buffer-first-based adaptation method. In this method, users will always consider filling a predefined buffer length (evaluated by the remaining playout time of the downloaded video segments). When the buffer length is larger than the predefined buffer length, video segments with higher bitrates will be requested. The buffer-first-based method avoids the challenge of estimating channel bandwidths, and stabilizes the buffer occupancy to ensure smooth video playback. However, such method usually requests video segments with lower quality than the qualityfirst-based method.

Meng et al. [21] proposed a proportional-integral-derivative (PID)-based rate adaption method for DASH. The performance of this method is overwhelming for channels with stable parameters, but it is difficult to determine the appropriate control parameters for the PID model when the channel state fluctuates. By considering the influence of the current requested video segment on the next requested video segment, Zhou et al. [22] and Bokani et al. [23] proposed a Markov decision process (MDP)-based rate adaptation methods, respectively, where the request process for a video segment is

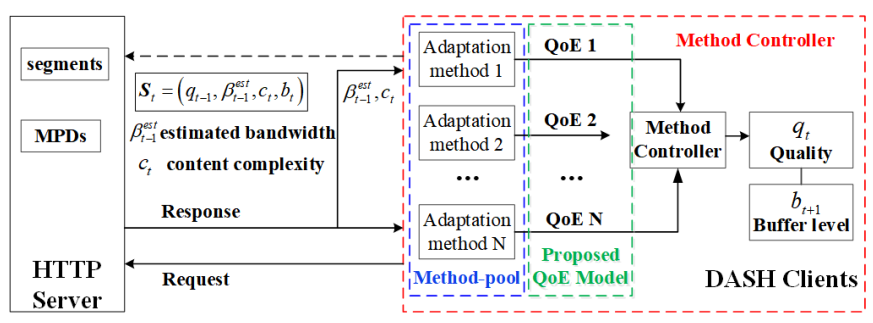

Fig. 2. Proposed ensemble learning based rate adaptation framework for DASH.

considered to be an MDP. To maximize the QoE, Martín et al. [24] proposed a Q-Learning-based rate adaptation method based on the Markov process assumption, in which the requested video segments can be dynamically adjusted based on the perceived network state. In spite of the pretty good performance of the Q-Learning based method, it needs a long time to converge. To overcome this limitation, Claeys et al. [25] proposed an HTTP Adaptive Streaming client based on Frequency Adjusted Q-Learning (FAQ). They simplified the MDP model and improved the learning rate of the process. However, this simplification leads to suboptimal streaming performance. In other words, there is a tradeoff between convergence speed and learning accuracy. Chiariotti et al. [17] proposed an online learning-based rate adaptation method. In this method, the developed learning process exploits a parallel learning technique that improves the learning rate and limits sub-optimal choices, leading to a fast and accurate learning process that converges to the best state rapidly. Unfortunately, it is hard to make the best request decision when the channel parameters frequently change.

All the methods are expected to achieve the optimal rate adaptation and guarantee the best user $Q o E$ by only using one model. Due to the uncertainty of network throughput/bandwidth requirements, a single model is unlikely to perform well at all times. Therefore, we propose an ensemble rate adaptation framework for DASH.

\section{PRoposed ENSEMble LEARNING-BASED FRAMEWORK}

\section{A. Overview of the Proposed Framework}

As shown in Fig. 2, the proposed framework is composed of two modules: the method pool and the method controller. In the method pool, several rate adaptation methods are integrated. At each decision time, the method that can achieve the best $Q o E$ is chosen to determine the bitrate of the requested video segment. It is also worth pointing out the remaining unselected methods will also update their parameters based on the actual system information (e.g., estimated throughputs, current buffer level, video complexity, etc.) to simulate their request procedure and calculate their corresponding estimated QoEs. The method controller will finally decide which method is the best according to their QoEs. In the following sections, we will introduce the method pool, the method controller, and the proposed QoE model in detail.

For clear representation, a summary of notations is given in TABLE I. 
TABLE I

NOTATIONS

\begin{tabular}{|c|c|c|c|}
\hline Notation & Meaning & Notation & Meaning \\
\hline$b_{k 1}, b_{k 2}$ & $\begin{array}{l}\text { buffer threshold used in the PD controller- } \\
\text { based method }\end{array}$ & $\bar{Q}$ & $\begin{array}{l}\text { the average quality of a video } \\
\text { segment }\end{array}$ \\
\hline$k_{p}, k_{d}$ & $\begin{array}{l}\text { the two parameters for the PD controller - } \\
\text { based method }\end{array}$ & $P_{Q V}$ & $\begin{array}{l}\text { the quality variations of the received } \\
\text { video segments }\end{array}$ \\
\hline$b_{t}$ & buffer length at time $t$ & $P_{I P D}$ & initial playout delay \\
\hline$r_{t-1}, r_{t}$ & $\begin{array}{l}\text { the bitrate of the previous requested and the } \\
\text { video segment that will be downloaded }\end{array}$ & $P_{R B}$ & the re-buffering duration \\
\hline$D_{t-1}$ & $\begin{array}{l}\text { the downloading time of the previous } \\
\text { segment }\end{array}$ & $q_{t-1}, q_{t}$ & $\begin{array}{l}\text { the quality of the previously received } \\
\text { and the current downloaded video } \\
\text { segment }\end{array}$ \\
\hline$\beta_{t-1}^{e s t}$ & the estimated bandwidth of time $t$ & $c_{t}$ & $\begin{array}{l}\text { the content complexity of the video } \\
\text { segment to be downloaded }\end{array}$ \\
\hline$\beta_{t}$ & the actual bandwidth of time $t$ & $b_{t}$ & the current buffer length \\
\hline$T$ & the playout time of a video segment & $D_{t}^{e s t}$ & $\begin{array}{l}\text { the estimated download time of the } \\
\text { current requested video segment }\end{array}$ \\
\hline$\overline{R_{w d}}$ & $\begin{array}{l}\text { the average reward of the previous } N \\
\text { segments }\end{array}$ & $b_{t+1}^{e s t}$ & $\begin{array}{l}\text { the estimated buffer length of time } \\
t+1\end{array}$ \\
\hline$R_{w d}^{t}$ & the instantaneous reward at time $t$ & $P_{B}$ & buffer stabilization factor \\
\hline$p_{a r w d, i}$ & the average reward of method $i$ & $\begin{array}{c}\omega_{1}, \omega_{2} \\
\omega_{3}\end{array}$ & $\begin{array}{l}\text { parameters of the proposed QoE } \\
\text { model }\end{array}$ \\
\hline$p_{\text {ratio }, i}$ & $\begin{array}{l}\text { the percentage that the reward of the method } \\
i \text { achieves the maximum in the previous } N \\
\text { segments }\end{array}$ & $P$ & transition matrix of Markov channel \\
\hline
\end{tabular}

\section{B. Method Pool}

In this part, we introduce three methods as components of the method pool module of the proposed ensemble rate adaptation framework, i.e., a rate-based method [3], a proportion differentiation (PD) controller-based method [18], and an online learning-based method [17]. It is worth pointing out that we select these three methods as a typical implementation example, and other methods can also be integrated into the method pool.

\section{(i) The rate-based method}

The rate-based method will request the video segment whose bitrate is closest to the estimated bandwidth [3]. As the rate-based method is simple yet effective to some extent, it has been extensively used in many adaptive streaming systems, e.g. Microsoft Smooth Streaming [38-39], Adobe HTTP Dynamic Streaming [40] and Apple's HTTP Live Streaming [41].

\section{(ii) The PD controller-based adaptation method}

In this method, two buffer thresholds, $b_{k 1}$ and $b_{k 2}$, are defined to restrict fluctuations of the user buffer. First, the video segment with the lowest bitrate is downloaded. When the buffer length at time $t$ (denoted by $b_{t}$ ) is larger than $b_{k 1}$ and smaller than $b_{k 2}$, the requested bitrate will remain unchanged. When the $b_{t}$ is less than $b_{k 1}$, the requested bitrate will be calculated as

$$
r_{t}=r_{t-1}+\frac{1}{T} \times \beta_{t-1}^{e s t}\left[k_{p} \times\left(b_{t}-b_{k 1}\right)+k_{d} \times \frac{T-D_{t-1}}{D_{t-1}}\right],
$$

whereas, when the $b_{t}$ is larger than $b_{k 2}$, the requested bitrate will be calculated as

$$
r_{t}=r_{t-1}+\frac{1}{T} \times \beta_{t-1}^{e s t}\left[k_{p} \times\left(b_{t}-b_{k 2}\right)+k_{d} \times \frac{T-D_{t-1}}{D_{t-1}}\right],
$$

where $r_{t-1}$ is the bitrate of the previous requested video segment; $r_{t}$ is the bitrate of the video segment that will be downloaded; $D_{t-1}$ is the downloading time of the previous segment; $\beta_{t-1}^{\text {est }}$ is the estimated bandwidth; $T$ is the playout time of a video segment, e.g., 2 seconds; $k_{p}$ and $k_{d}$ are the two parameters of the PD controller. Based on [18], the two parameters $k_{d}$ and $k_{p}$ can be set as

$$
\left\{\begin{array}{l}
k_{p}=\eta \times \sqrt{T^{2}-k_{d}^{2}}, \\
\eta \geq \frac{1}{T} \sqrt{\frac{T+k_{d}}{T-k_{d}}} \ln \frac{20 T}{T+k_{d}}, \\
0<k_{d}<T .
\end{array}\right.
$$

\section{(iii) The online learning-based adaptation method}

By assuming that the channel bandwidth experienced by the users as well as the video characteristic variation follows a Markov model, the online learning-based method [17] formulates the optimal action selection problem as a Markov Decision Process (MDP). The system dynamics in the MDP model are a priori unknown and thus learned through a Reinforcement Learning technique [17], in which the $Q o E$ of users is dynamically maximized.

At each decision time, the method controller of our framework will select a method from the method pool to request a video segment so that the $Q o E$ of users can be maximized.

\section{Method Controller}

As aforementioned, there are multiple rate adaptation methods in the method pool. For the first several decision times (each decision time corresponds to a video segment), a default method is selected. Then, at the subsequent decision times, the method controller selects one method to request a video segment. At the same time, the remaining unselected methods will also perform a "virtual request" based on the estimated bandwidth and then update their "virtual buffers". The method controller will always check the states of all the rate adaptation methods and select the one that can achieve the highest $Q o E$. In order to select an appropriate rate adaptation method, we also design two strategies to enable the controller to effectively switch from one method to another, i.e., InstAnt Method Switching (IAMS), and InterMittent Method Switching (IMMS).

For the IAMS strategy shown in Fig. 3, the method controller will always check the average reward (denoted as $\overline{R_{w d}}$ ) of the previous $N$ decision times (segments) of all the methods in the method pool, and then select the method that can achieve the maximum average reward to request a video segment at the current decision time. Note that the reward of a method in the previous decision time may be "virtual" or "actual", which depends on whether the method was selected at that decision time or not.

The IAMS strategy may result in frequent method switching. Therefore, we also propose the IMMS strategy as shown in Fig. 4. In this method, the method controller will intermittently check the product of the average reward of each method and the ratio that the reward of a method achieves the maximum in the previous $N$ segments:

$$
p_{\text {product }, i}=p_{\text {arwd }, i} \times p_{\text {ratio }, i},
$$




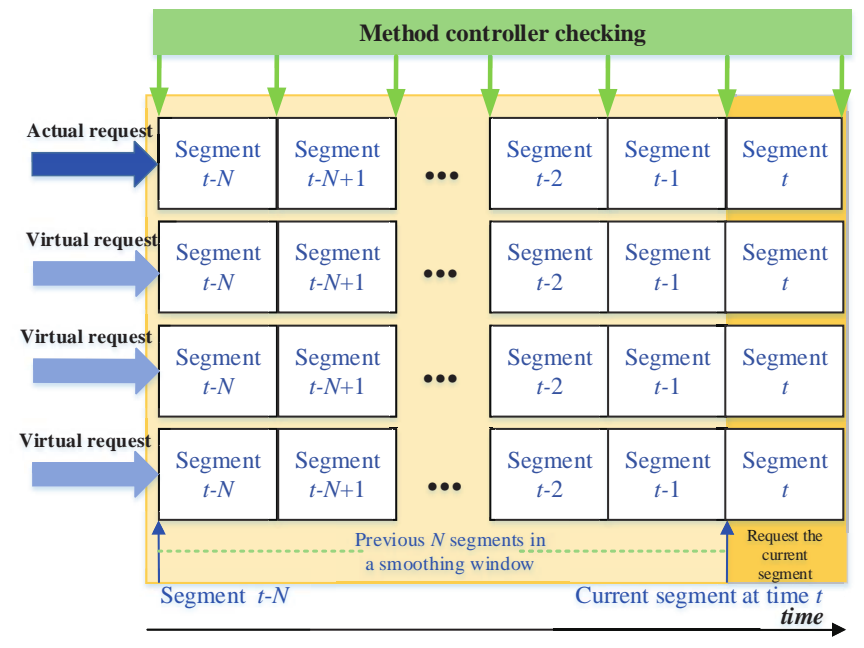

Fig. 3. Instant method switching.

where $p_{\text {arwd, } i}$ denotes the average reward of method $i$, and $p_{\text {ratio, } i}$ is the percentage that the reward of the method $i$ achieves the maximum in the previous $N$ segments. Then, the method controller will select the method that can achieve the maximum product to request a video segment for the next $N$ segments.

\section{The proposed QoE Model}

The user $Q o E$ must be considered and estimated well when designing rate adaptation methods. Typically, by taking the visual memory [42] into into account, the user $Q o E$ can be estimated based on four factors [6-7] [17] [24-25] [43-45]: the average quality level, the quality variations during the video playout, the video freezes, and the startup delay. By investigating the existing $Q o E$ models [6-7] [24-25] [43-49], the $Q o E$ model can be written as

$$
Q o E=\bar{Q}-\omega_{0} P_{I P D}-\omega_{1} P_{Q V}-\omega_{2} P_{R B},
$$

where $\bar{Q}$ and $P_{Q V}$ denote the average quality and the quality variations of the received video segments, respectively; $P_{I P D}$ and $P_{R B}$ denote the initial playout delay and the re-buffering time, respectively; $\omega_{0}, \omega_{1}$, and $\omega_{2}$ are the model parameters.

Based on Eq.(5), to estimate the user LT-QoE, the system state that can be derived from $\bar{Q}, P_{Q V}$ and $P_{I P D}$ must be available. In the proposed rate adaptation framework, the system state at time $t$ is defined as $\boldsymbol{S}_{t}=\left(q_{t-1}, \beta_{t-1}^{e s t}, c_{t}, b_{t}\right)$, where $q_{t-1}$ is the quality of the previously received video segment, $\beta_{t-1}^{\text {est }}$ is the estimated bandwidth, $c_{t}$ is the content complexity of the video segment to be downloaded, and $b_{t}$ is the current buffer length. Based on the defined system state, the average quality $\bar{Q}$ is replaced with the quality of the video segment that may be downloaded at the decision time $t$, i.e. $q_{t}$, while the quality variations $P_{Q V}$ are simplified as $\left|q_{t}-q_{t-1}\right|$. The re-buffering time $P_{R B}$ is represented with $\max \left\{\left(D_{t}^{\text {est }}-b_{t}\right), 0\right\}$ where $D_{t}^{\text {est }}$ is the estimated download time of the video segment requested at time $t$ :

$$
D_{t}^{e s t}=r_{t} \times T / \beta_{t-1}^{e s t},
$$

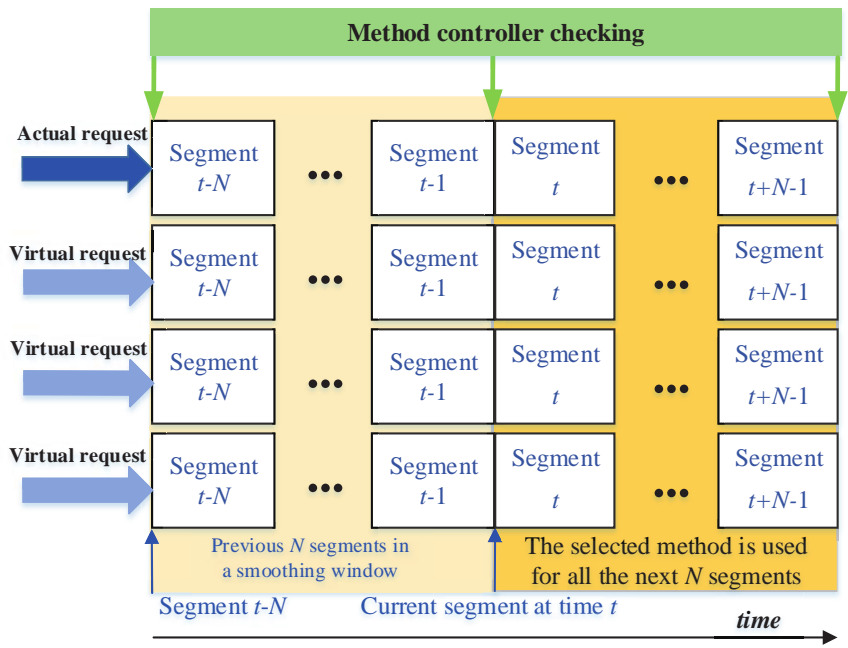

Fig. 4. Intermittent method switching.

To stabilize the buffer length to a predefined value $\left(b_{0}\right)$, we add another item $\left(P_{B}\right)$ in the $Q o E$ model, which is expressed as

$$
P_{B}=g\left(b_{t+1}^{e s t}-b_{0}\right) \times\left|b_{t+1}^{e s t}-b_{0}\right|,
$$

where

$$
b_{t+1}^{e s t}=b_{t}+T-D_{t}^{e s t},
$$

is the estimated buffer length of time $t+1$, and $g(\cdot)$ is a function defined as:

$$
g(\cdot)= \begin{cases}-1 & b_{t+1}^{e s t}-b_{0}<0 \\ -0.25 & b_{t+1}^{e s t}-b_{0} \geq 0\end{cases}
$$

When $b_{t+1}^{e s t}$ is smaller than $b_{0}$, the risk of playout interruption (re-buffering) is large. Thus, we use a large penalty coefficient of value -1 for $\left|b_{t+1}^{\text {est }}-b_{0}\right|$ to select video segments with lower bitrates. When $b_{t+1}^{\text {est }}$ is larger than $b_{0}$, the risk of playout interruption (re-buffering) is small, and we use a small penalty coefficient of value -0.25 for $\left|b_{t+1}^{\text {est }}-b_{0}\right|$ to select video segments with higher bitrates. Since the start up delays are manually set as a fixed value (e.g. $2 \mathrm{~s}, 5 \mathrm{~s}$, etc.), $\omega_{0}$ is usually set to be zero. Finally, the user $Q o E$ (we also call it as the reward at time $t, R_{w d}^{t}$ ) model used in the proposed rate adaptation framework is formulated as

$$
\begin{aligned}
R_{w d}^{t}= & Q o E=q_{t}-\omega_{1} \times\left|q_{t}-q_{t-1}\right|-\omega_{2} \\
& \times \max \left\{\left(D_{t}^{e s t}-b_{t}\right), 0\right\}-\omega_{3} \times P_{B},
\end{aligned}
$$

where $\omega_{1}, \omega_{2}$, and $\omega_{3}$ are the model parameters.

\section{Simulation Results AND Analysis}

\section{A. Simulation Settings}

The experiments were conducted over the video sequences taken from the EvalVid CIF video trace reference database. The quality levels, complexity level, and the corresponding set of available bitrates are described in TABLE II. Note the complexity levels are predefined for all the video traces based on [17]. The Structural SIMilarity index (SSIM) [50] is used as the quality metric for video segments. The value of SSIM 
TABLE II

QUALITY LEVELS AND CORRESPONDING BITRATES

\begin{tabular}{|c|c|c|c|c|c|c|c|}
\hline $\begin{array}{c}\text { Complexity } \\
\text { Level }\end{array}$ & $\begin{array}{l}\text { Quality } \\
\text { Level }\end{array}$ & $\begin{array}{l}\text { Bitrate } \\
(\mathrm{Mb} / \mathrm{s})\end{array}$ & SSIM & $\begin{array}{c}\text { Complexity } \\
\text { Level }\end{array}$ & $\begin{array}{c}\text { Quality } \\
\text { Level }\end{array}$ & $\begin{array}{l}\text { Bitrate } \\
(\mathrm{Mb} / \mathrm{s})\end{array}$ & SSIM \\
\hline \multirow{8}{*}{1} & 1 & 10 & 1 & \multirow[t]{8}{*}{ - } & 1 & 10 & 1 \\
\hline & 2 & 6 & 0.9976 & & 2 & 6 & 0.9985 \\
\hline & 3 & 4 & 0.9955 & & 3 & 4 & 0.9966 \\
\hline & 4 & 3 & 0.9940 & & 4 & 3 & 0.9949 \\
\hline & 5 & 2 & 0.9921 & & 5 & 2 & 0.9921 \\
\hline & 6 & 1 & 0.9898 & & 6 & 1 & 0.9859 \\
\hline & 7 & 0.5 & 0.9875 & & 7 & 0.5 & 0.9758 \\
\hline & 8 & 0.3 & 0.9843 & & 8 & 0.3 & 0.9635 \\
\hline $\begin{array}{c}\text { Complexity } \\
\text { Level }\end{array}$ & $\begin{array}{r}\text { Quality } \\
\text { Level }\end{array}$ & $\begin{array}{l}\text { Bitrate } \\
(\mathrm{Mb} / \mathrm{s})\end{array}$ & SSIM & $\begin{array}{c}\text { Complexity } \\
\text { Level }\end{array}$ & $\begin{array}{c}\text { Quality } \\
\text { Level }\end{array}$ & $\begin{array}{l}\text { Bitrate } \\
\text { (Mb/s) }\end{array}$ & SSIM \\
\hline \multirow{8}{*}{3} & 1 & 10 & 1 & \multirow{8}{*}{4} & 1 & 10 & 1 \\
\hline & 2 & 6 & 0.9938 & & 2 & 6 & 0.9988 \\
\hline & 3 & 4 & 0.9850 & & 3 & 4 & 0.9965 \\
\hline & 4 & 3 & 0.9777 & & 4 & 3 & 0.9938 \\
\hline & 5 & 2 & 0.9666 & & 5 & 2 & 0.9881 \\
\hline & 6 & 1 & 0.9479 & & 6 & 1 & 0.9771 \\
\hline & 7 & 0.5 & 0.9321 & & 7 & 0.5 & 0.9436 \\
\hline & 8 & 0.3 & 0.9228 & & 8 & 0.3 & 0.9127 \\
\hline $\begin{array}{c}\text { Complexity } \\
\text { Level }\end{array}$ & $\begin{array}{c}\text { Quality } \\
\text { Level }\end{array}$ & $\begin{array}{l}\text { Bitrate } \\
(\mathrm{Mb} / \mathrm{s})\end{array}$ & SSIM & & \multirow{9}{*}{ NA } & & \\
\hline \multirow[t]{8}{*}{ 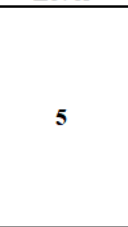 } & 1 & 10 & 1 & & & & \\
\hline & 2 & 6 & 0.9984 & & & & \\
\hline & 3 & 4 & 0.9933 & & & & \\
\hline & 4 & 3 & 0.9864 & & & & \\
\hline & 5 & 2 & 0.9705 & & & & \\
\hline & 6 & 1 & 0.9221 & & & & \\
\hline & 7 & 0.5 & 0.8415 & & & & \\
\hline & 8 & 0.3 & 0.7584 & & & & \\
\hline
\end{tabular}

ranges from 0 to 1 , and when the SSIM value is smaller than 0.9 , we consider the quality to be poor.

The parameter $\omega_{1}$ in Eq. (10) was set to 2 to get a good tradeoff between the average quality and the quality switches. Parameters $\omega_{2}, \omega_{3}$, and $b_{0}$ are used to adjust the buffer penalty in Eq. (10). We then empirically set $b_{0}=8 \mathrm{~s}, \omega_{2}=50$, and $\omega_{3}=0.0001$ to achieve a sufficiently low probability of rebuffering events and yet allow the framework to efficiently use the buffer in order to decrease quality variations. Because of the common choice of DASH controllers, we also limit the largest buffer length to be $b_{\max }=20 \mathrm{~s}$. Finally, the exponential discount factor $\gamma$ was set as 0.9 to achieve a good tradeoff between the immediate and future rewards.

In simulation, four kinds of channel environments were considered: the constant channel, the short-term fluctuating channel, the long-term fluctuating channel, and the Markov channel (see Fig. 5). For the Markov channel [22] [51] [52], assuming that there are $k$ states, the following $k \times k$ transition matrix $\boldsymbol{P}$ is set as

$$
\boldsymbol{P}=\left(\begin{array}{ccc}
p_{11} & \cdots & p_{1 k} \\
\vdots & \ddots & \vdots \\
p_{k 1} & \cdots & p_{k k}
\end{array}\right),
$$

where $p_{i j}$ denotes the transition probability from state $i$ to state $j$ :

$$
p_{i j}= \begin{cases}2 p / 3, & |i-j|=1, \\ p / 3, & |i-j|=2 .\end{cases}
$$

A large $p$ corresponds to a highly dynamic channel. By varying $p$, we can simulate different dynamic channels. In the simulations, we set $p$ to $0,0.25$ and 0.5 [17]. To verify the performance of the proposed ensemble rate adaptation framework, we have compared it with other methods using MATLAB simulation.
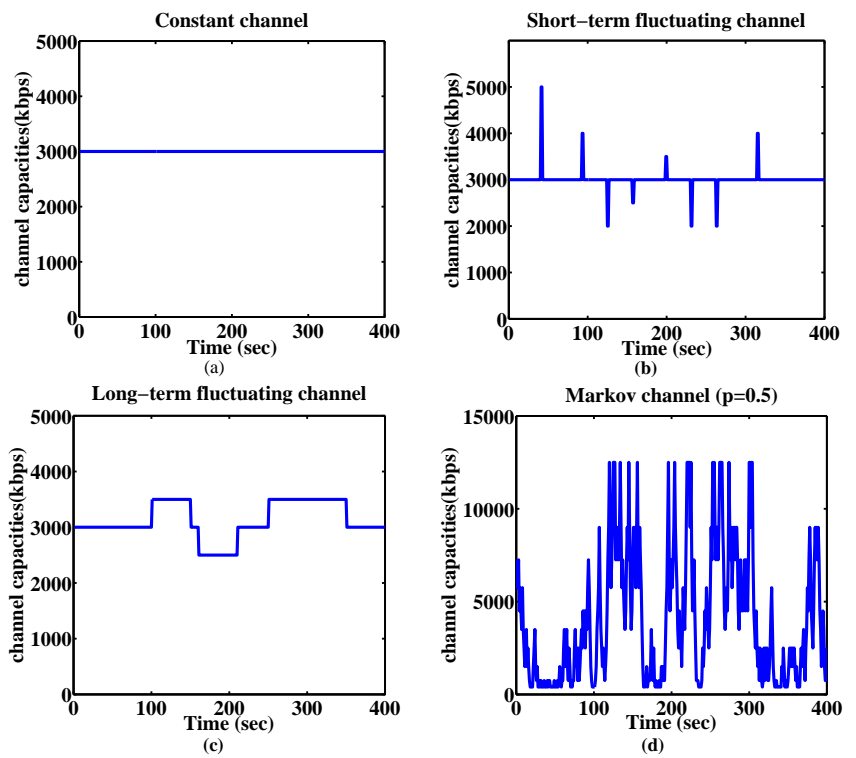

Fig. 5. Four kinds of channel environments.
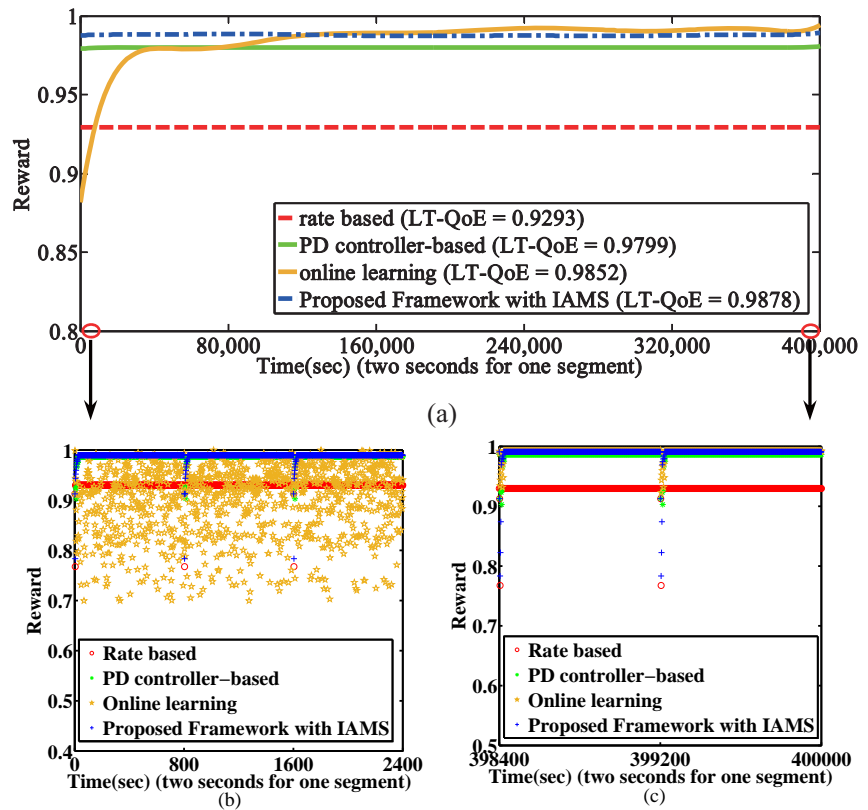

(c)

Fig. 6. Rewards and LT-QoEs comparison under the constant channel.

\section{B. Simulation Results}

\section{(i) Results of the proposed framework with IAMS}

First, we compared the methods in a static scenario in which both the channel capacity $\left(\beta_{t}^{e s t}=3 \mathrm{Mbps}\right)$ and video complexity $(c=4)$ are constant. Then, we performed simulations on the other three channels: the short-term fluctuating channel, long-term fluctuating channel, and Markov channel, as shown in Figs. 5 (b), (c), and (d), respectively. For the proposed framework (denoted by Proposed Framework with IAMS), the method controller will check the average reward denoted as $\left(\overline{R_{w d}}\right)$ of the previous $N_{I A M S}$ (that was empirically set to be 2) decision times (segments) of all the methods in the method pool. 

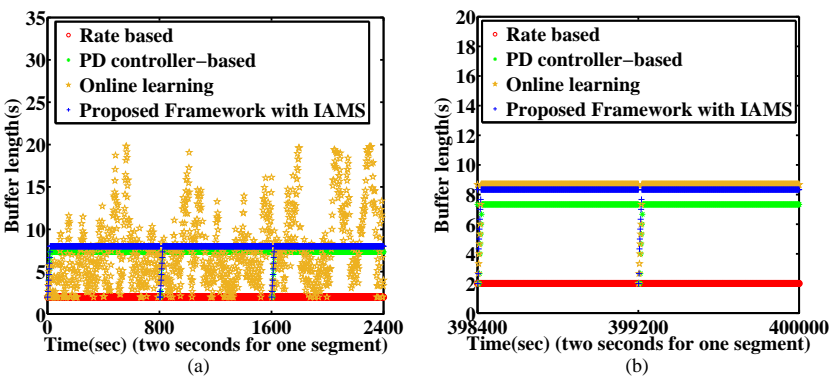

Fig. 7. Buffer length comparison under the constant channel.
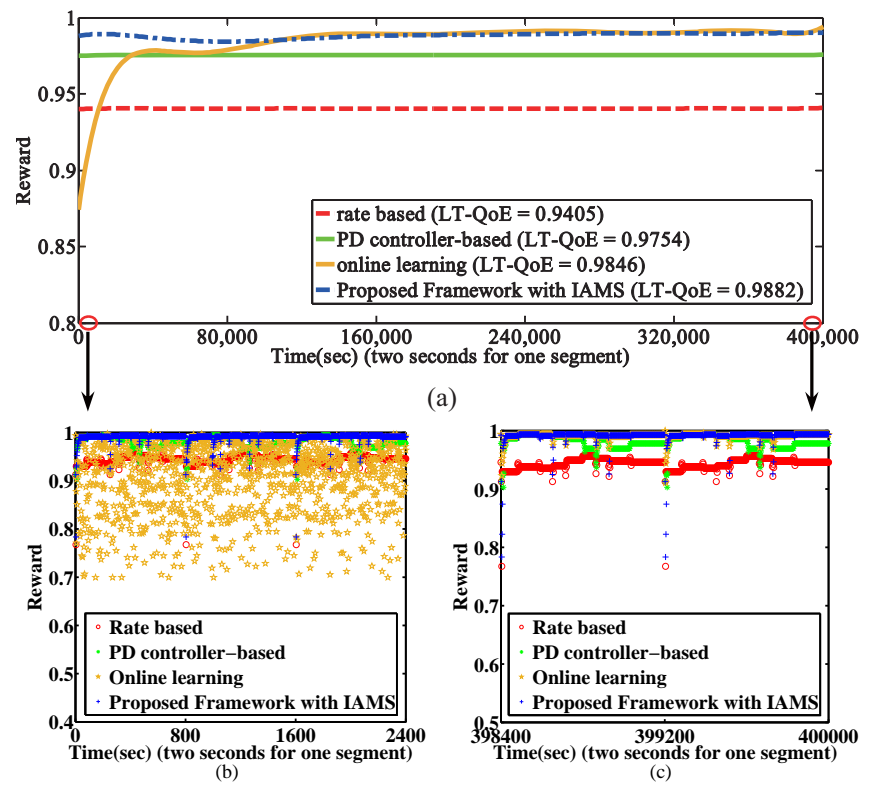

Fig. 8. Rewards and LT-QoEs comparison under the short-term fluctuating channel.

For the constant channel, the reward and buffer length of each method are compared in Figs. 6 and 7, respectively. As the proposed framework will select a rate adaptation method at each decision time from the method pool, the instant reward of the proposed framework would be similar with the selected method, especially at the beginning of the request. Note that Fig. 6 (a) shows the nine order polynomial fitting curves of the rewards of the four methods to show the comparison results clearly. Therefore, there is a gap between the proposed framework and the other methods at the beginning of the request.
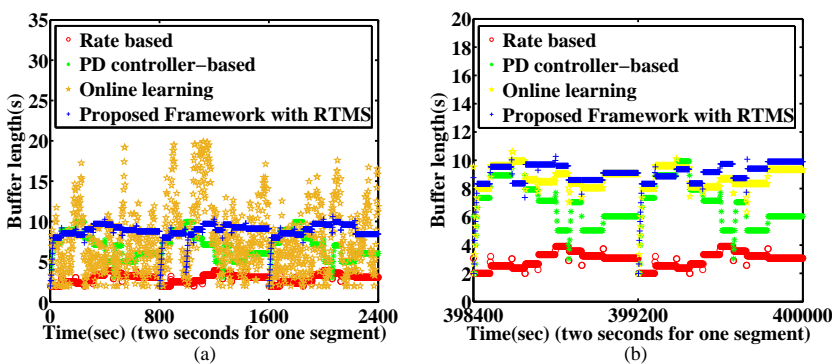

Fig. 9. Buffer length comparison under the short-term fluctuating channel.
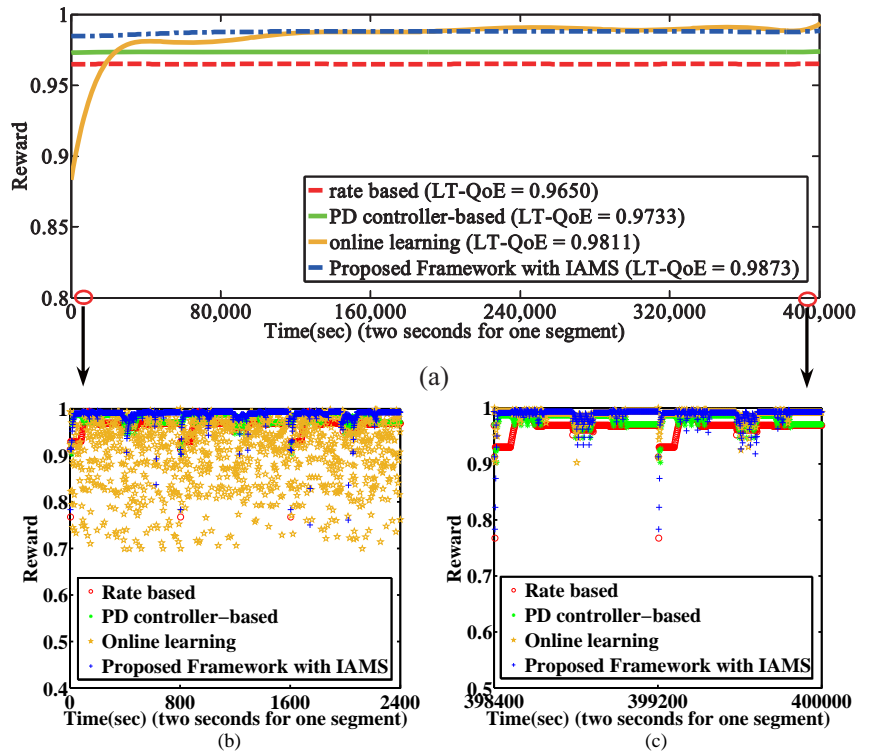

Fig. 10. Rewards and LT-QoEs comparison under the long-term fluctuating channel.
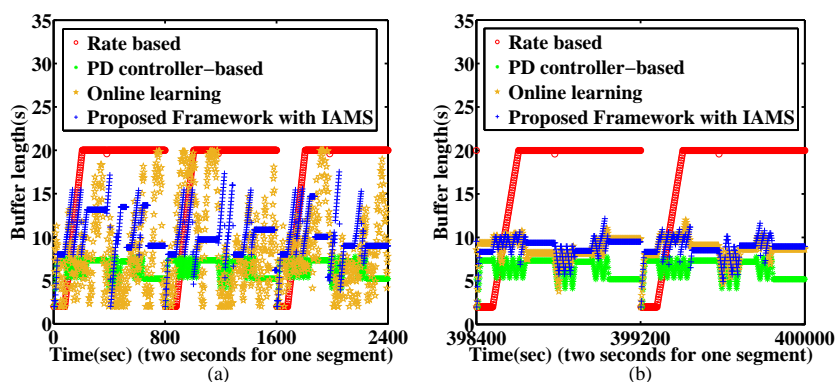

Fig. 11. Buffer length comparison under the long-term fluctuating channel.
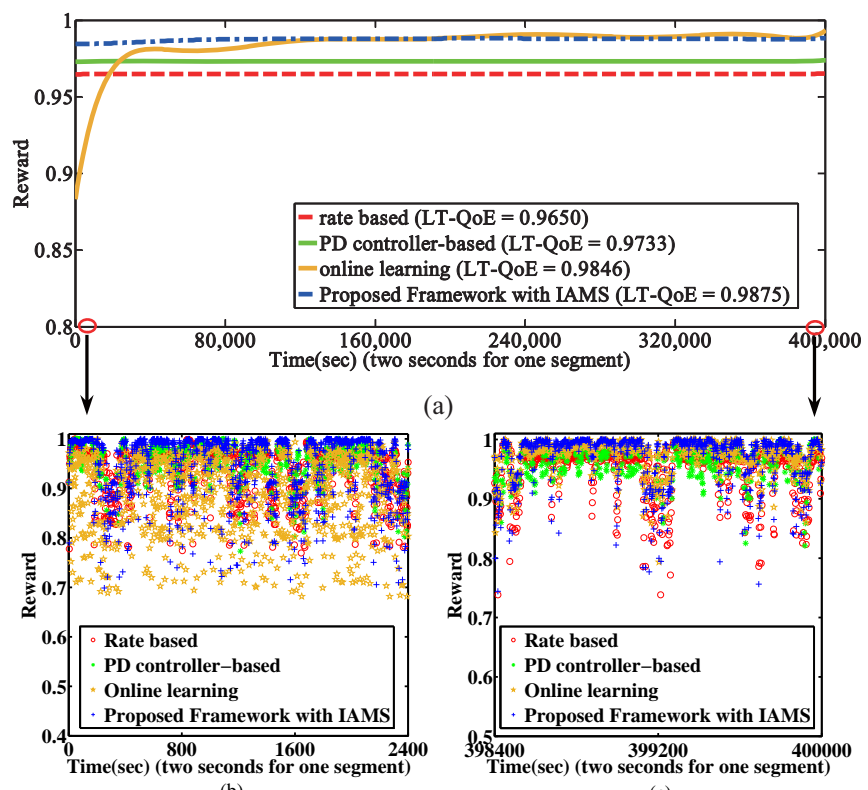

(b)

Fig. 12. Rewards and LT-QoEs comparison under the Markov channel ( $p=$ 0.5 ). 

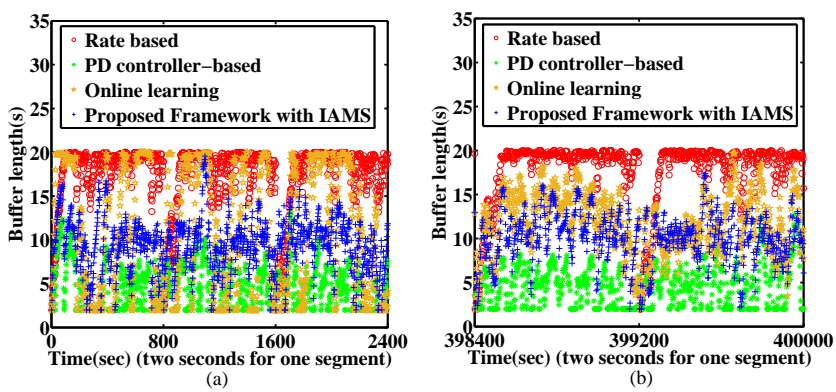

Fig. 13. Buffer length comparison under the Markov channel $(p=0.5)$.

However, from the detailed rewards shown in Fig. 6(b), we can see that the rewards of the proposed framework are similar to either the rate-based method or the PD controller based method at the beginning of the request. In the simulation, since the online learning-based method needs a very long time to converge, 500 episodes (each episode includes 400 segments) were requested to achieve a comprehensive comparison. To investigate the detailed reward variation of the four methods, Figs. 6 (b) and (c) compare the rewards of the first 2400s and the last 1600s. Since the channel bandwidth is constant $\left(\beta_{t}^{e s t}=3 \mathrm{Mbps}\right)$, the reward of the rate-based method is very stable, as shown in Fig. 6, whereas Fig. 7 shows that the buffer length of the rate-based method is small, because it always chooses the bitrate that matches the capacity exactly. For the online learning-based method, the reward is initially not convergent for the first 2400s in Fig. 6 (a). Accordingly, the buffer length also fluctuates greatly. When the online learning method converges, the average rewards and buffer lengths become stable. For the PD controller-based method, both the rewards and the buffer lengths are very stable, but the rewards are smaller than those of the converged online learning method. The Proposed Framework with IAMS can make use of the advantages of all the three methods. We can see that the rewards of the Proposed Framework with IAMS is more stable than those of the online learning-based method, especially at the initial requesting. This means that the proposed Proposed Framework with IAMS is more suitable for today's popular short video applications than the online learning method. From Fig. 7 (b), we can also observe that the buffer length of the Proposed Framework with IAMS fluctuates slowly, and is closer to the reference buffer length $\left(b_{0}=8 \mathrm{~s}\right)$ than the other three methods. Besides, the average rewards (denoted as $L T-Q o E \mathrm{~s}$ ), i.e., $\frac{1}{t_{\text {test }}} \sum_{t=t_{0}}^{t_{\text {test }}} R_{w d}^{t}$, of the four methods are also compared in Fig. 6. We can see that the LT-QoE of the Proposed Framework with IAMS is larger than each of the individual methods.

For the short-term fluctuating channel, long-term fluctuating channel, and the Markov channel $(p=0.5)$, similar results can also be observed in Figs. 8 to 13. The LT-QoEs of the Proposed Framework with IAMS are always the largest.

\section{(ii) Results of the proposed framework with IMMS}

Similar to part (i), we also compared the performance under the four kinds of channels (as shown in Fig. 5). The video complexity level is also set to be 4 . For the proposed framework (denoted by Proposed Framework with IMMS),

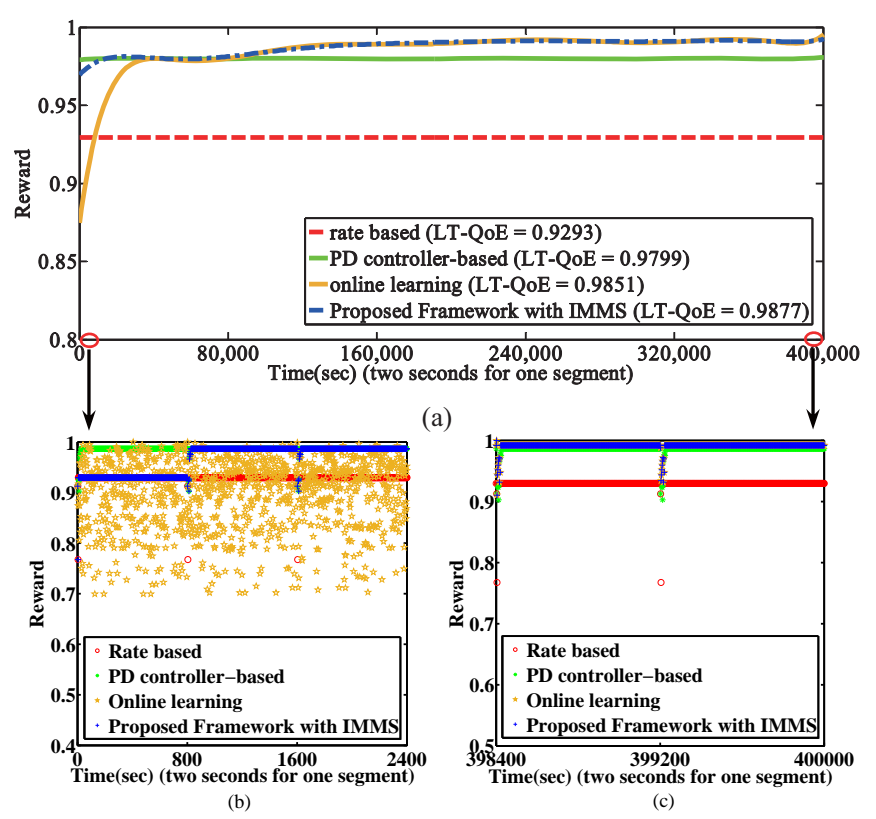

Fig. 14. Rewards and LT-QoEs comparison under the constant channel.
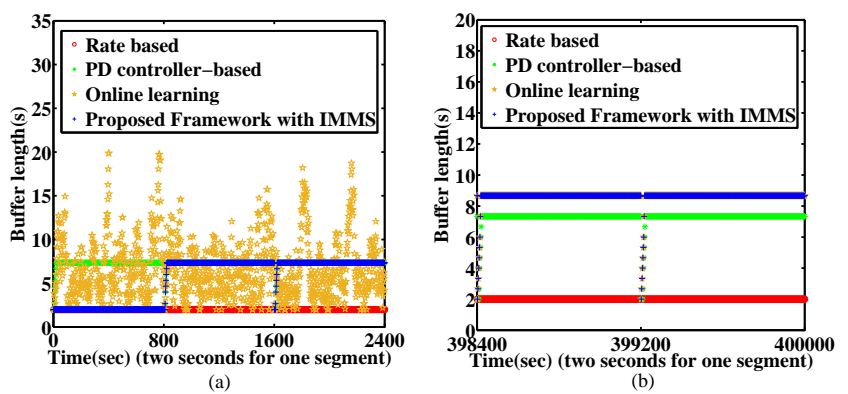

Fig. 15. Buffer length comparison under the constant channel.

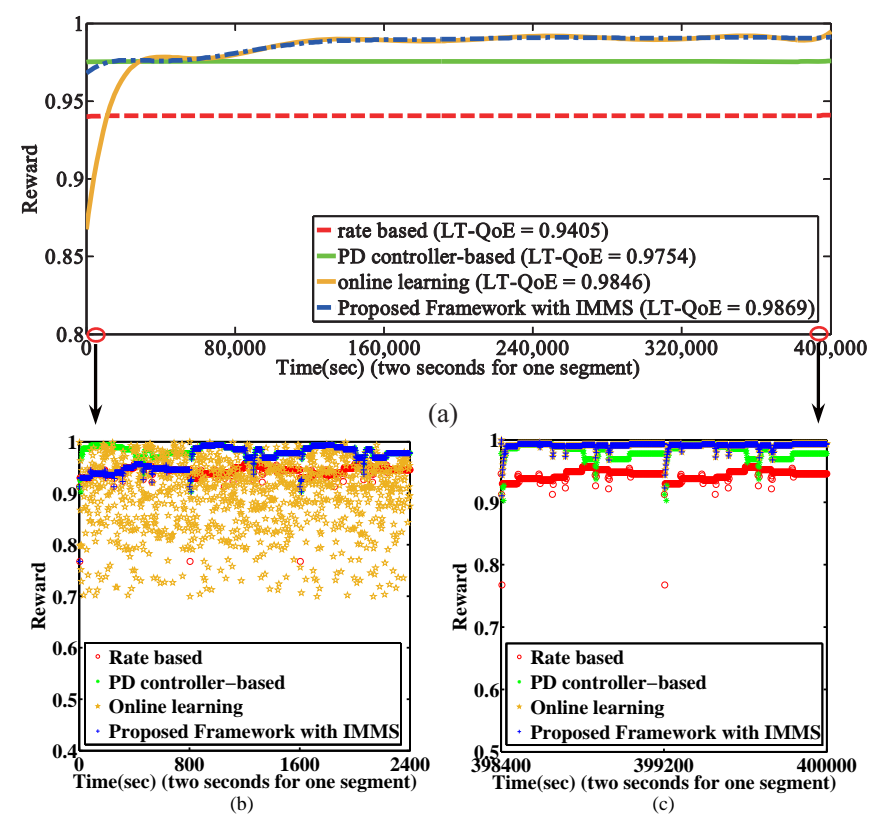

Fig. 16. Rewards and LT-QoEs comparison under the short-term fluctuating channel. 

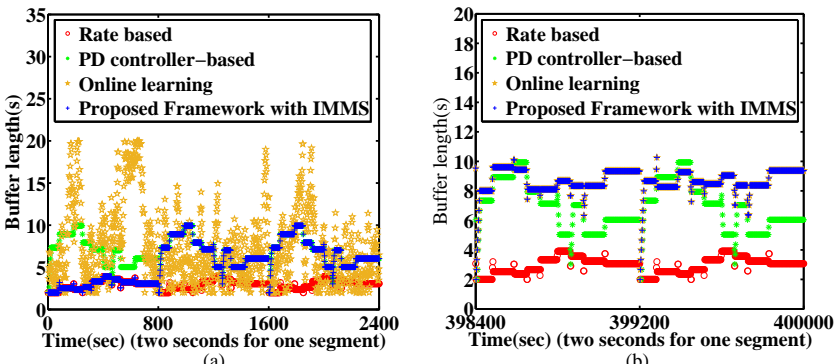

(a)

Fig. 17. Buffer length comparison under the short-term fluctuating channel.
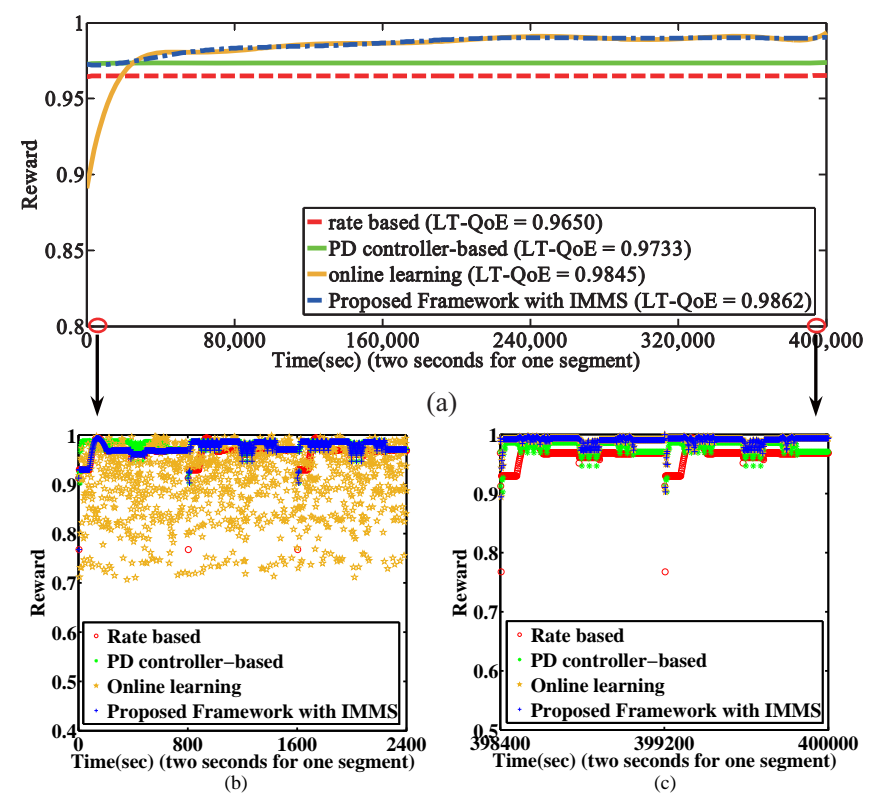

Fig. 18. Rewards and $L T-Q o E$ s comparison under the long-term fluctuating channel.

the method controller will check the product of the average reward (denoted as $\left(\overline{R_{w d}}\right)$ of each method and the ratio that the reward of a method achieves the maximum in the previous $N_{I M M S}$ segments $\left(N_{I M M S}=400\right.$, corresponding to an episode). The comparison results are shown in Figs. 14 to 21 for different kinds of channels. Since the rate-based method will be selected at the beginning of Proposed Framework with IMMS, the rewards and the buffer lengths of the Proposed Framework with IMMS are the same with those of the ratebased method for the first episode. From those figures, we can observe that the LT-QoEs of the Proposed Framework with IMMS are still the largest.

\section{(iii) Evaluation of the adaptation ability}

In order to further verify the adaptation ability of the proposed frameworks (both IAMS and IMMS), we first considered the case that the channel environment changes abruptly. In this simulation, there are 24000 video segments (corresponding to 600 episodes) to be requested. For the first 300 episodes (video complexity level is 4), a stable channel with a bandwidth of $3000 \mathrm{kbps}$ is simulated, while for the last 300 episodes, the channel environment changes abruptly to a Markov channel with $p=0.5$. Fig. 22 shows the fitted
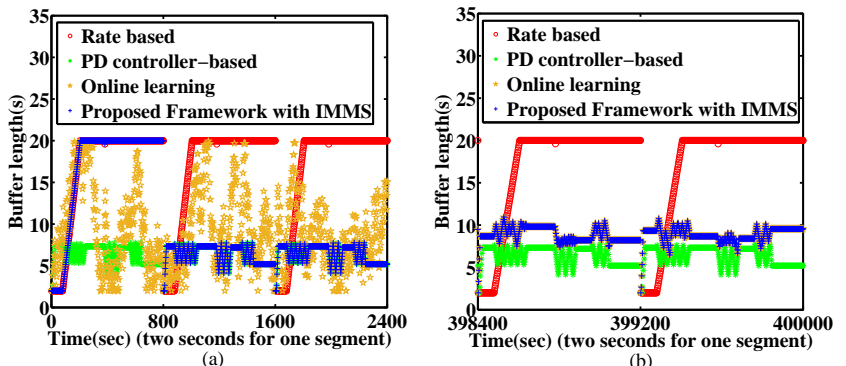

(a)

Fig. 19. Buffer length comparison under the long-term fluctuating channel.

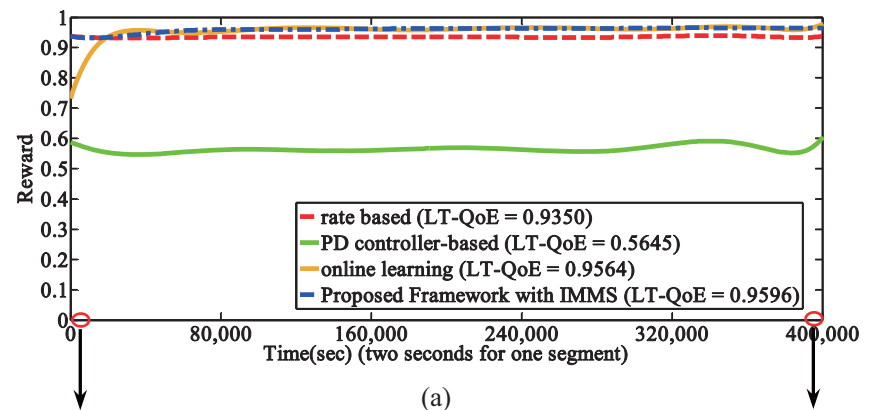

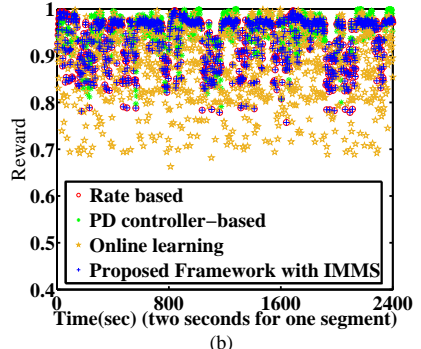

(a)

Fig. 20. Rewards and LT-QoEs comparison under the Markov channel ( $p=$ $0.5)$.

(also by a nine order polynomial fitting method) curves of the rewards for different rate adaptation methods. We can see that the rewards of the $P D$ controller-based method decline more seriously than the other methods. This is because the model parameters of the PD controller-based method cannot adapt the suddenly changed channel environment. We can also observe that the rewards of the online learning-based method and the proposed frameworks also suffer from sharp changes. But the $L T-Q o E$ of the proposed framework with IMMS is a little larger than that of the online learning-based method. To show
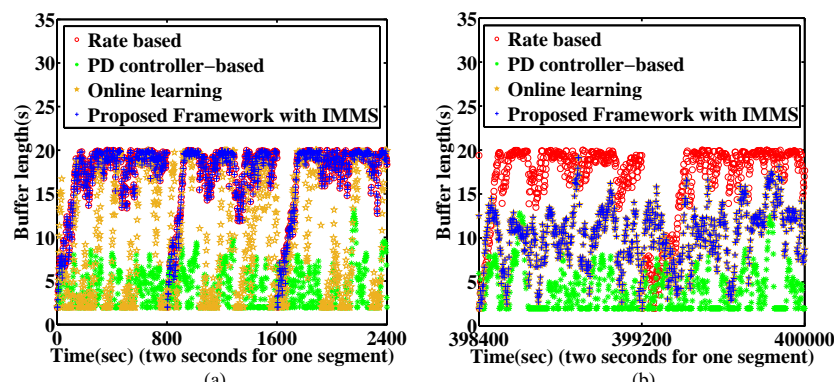

(b)
Fig. 21. Buffer length comparison under the Markov channel $(p=0.5)$. 


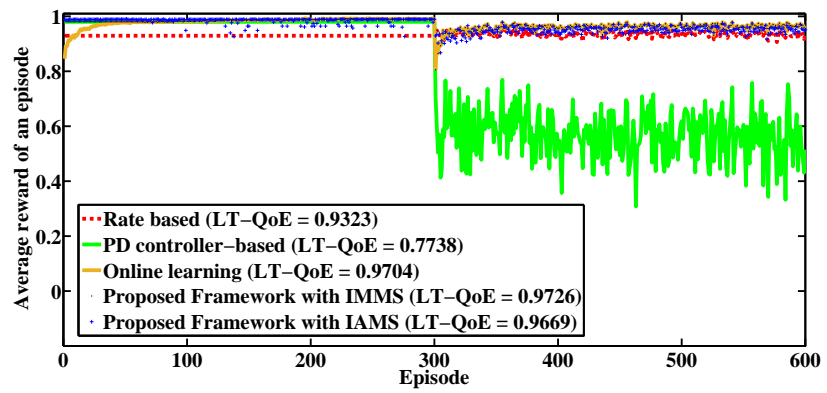

Fig. 22. Rewards comparison for 600 episodes with abrupt channel changes at the 300-th episode.

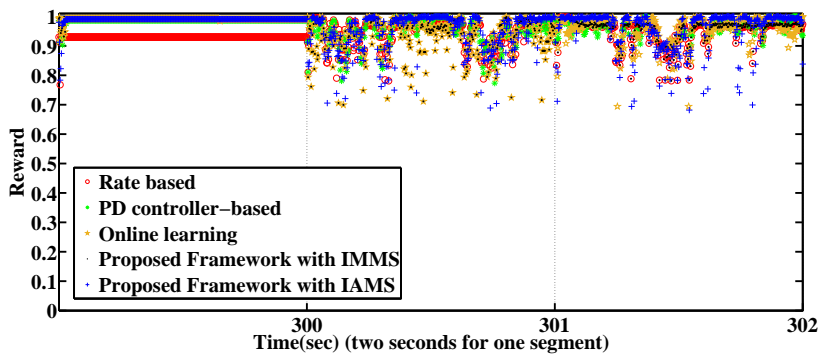

Fig. 23. Rewards comparison of the 300-th, 301-th, and the 302-th episodes.

the instantaneous rewards clearly at the changing time, Fig. 23 compares the detailed instantaneous rewards of different rate adaptation methods at the changing time.

Besides, the adaptation ability is also investigated on the video complexity variation. In this simulation, there are also 600 episodes to be requested. A Markov channel with $p=0.5$ is used. For the first 300 episodes, the complexity is 5 , while for the last 300-th episode, the video complexity level randomly changes from 5 to 1 . Fig. 24 shows the rewards of each episode for different methods. We see that the performance of the rate-based method is the worst since it cannot adapt to the Markov channel efficiently. Since the model parameters of the $P D$ controller-based method are not suitable, the performance of this method is also not very good. We can also see that the performances of the proposed frameworks are a little better than the online learning-based method.

Moreover, we also tested the dynamic case that both the

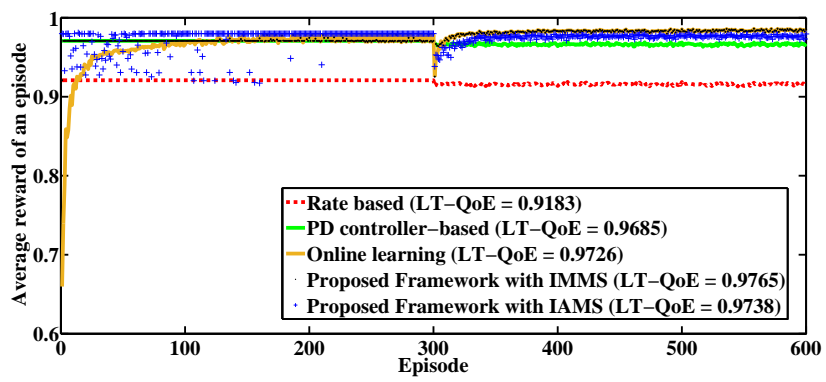

Fig. 24. Rewards comparison under the condition that the video complexity changes from a constant one $(c=5)$ to random values after the 300-th episode.

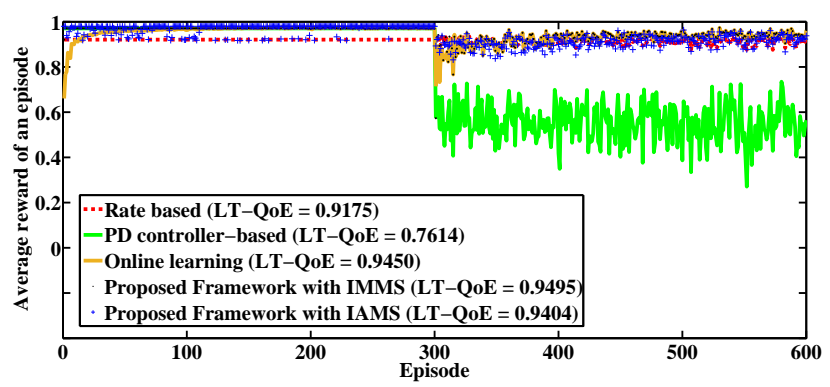

Fig. 25. Rewards comparison under the condition that both the channel state and the video complexity changes at the 300-th episode

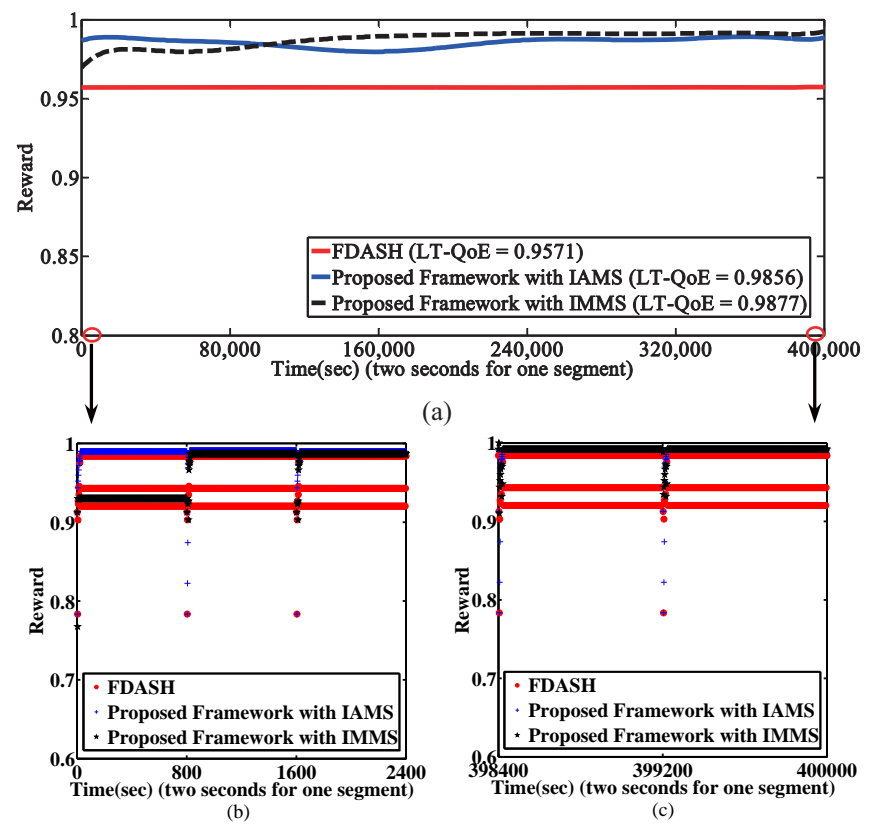

Fig. 26. Rewards and LT-QoEs comparisons under the constant channel.

channel environment and the video complexity level are changed. In this case, the channel environment is changed from a static channel to a Markov channel with parameter $p=0.5$ at the 300-th episode, while the video complexity is randomly changed for each segment after the 300-th episode, as shown in Fig. 25. We can see that the performance of the rate-based method is the worst. The performance of the $P D$ controllerbased method is also not good enough since it cannot adapt to the frequent fluctuations of channel environments. The online learning-based method can adapt to the complexity fluctuations to some extent, but the proposed frameworks (IMMS and IAMS) perform better. Also we should note that the $L T-Q o E$ of the online learning-based method is similar to that of the proposed frameworks as shown in Fig. 25. The reason is that the long term simulation time is propitious to the online learning-based method.

\section{(iv) Comparison with state-of-the-art methods}

In order to further validate the performance of the proposed frameworks (IMMS and IAMS), we have also compared them with a fuzzy logic-based rate adaptation method (denoted by FDASH ) [53] under different channels. Although the FDASH 

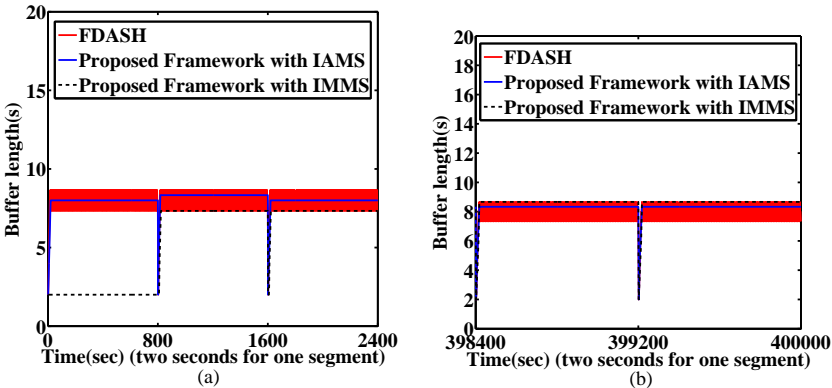

Fig. 27. Buffer length comparisons under the constant channel.

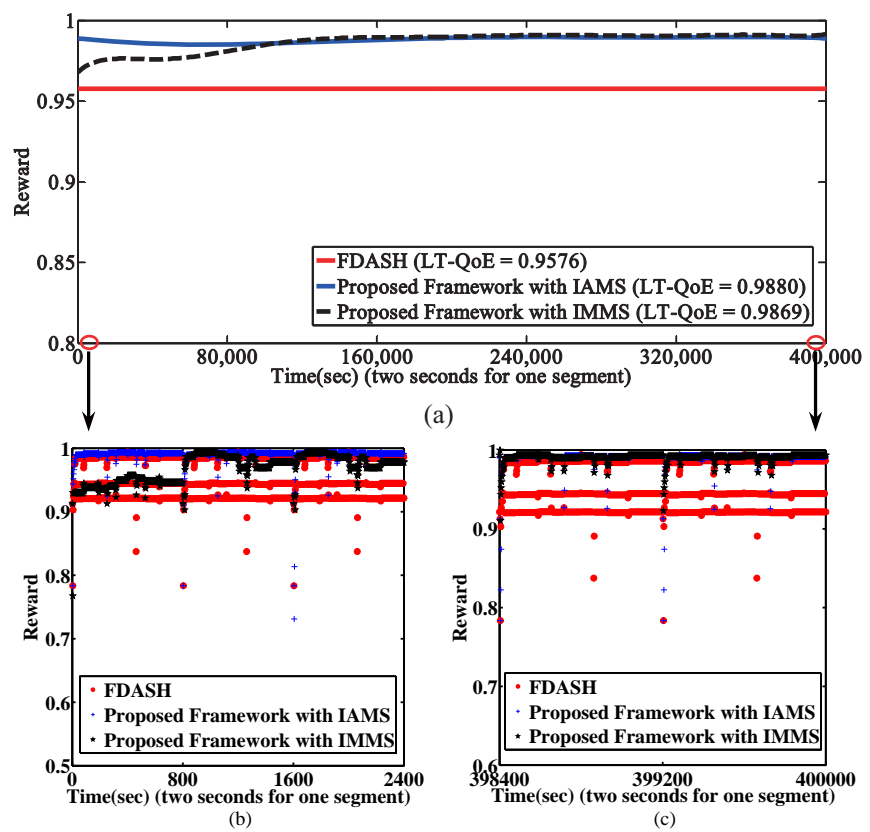

Fig. 28. Rewards and LT-QoEs comparisons under the short-term fluctuating channel.

method can also be integrated into the proposed frameworks, we should note that we only integrated the rate-based method, the PD controller-based method, and the online learningbased method into the IMMS and IAMS frameworks. For the constant channel, the rewards and LT-QoEs are compared in Fig. 26, while the corresponding buffer lengths are compared in Fig. 27. We can see that the buffer lengths of the FDASH are more stable than those of the proposed frameworks, but both the rewards and the $L T-Q o E$ of each of the proposed frameworks are larger than those of $\boldsymbol{F D A S H}$. Similar results can also be observed from Figs. 28-32, for the other channels.

\section{(v) Evaluation with commonly used QoE models}

Lastly, we evaluate the performance of the rate-based method, the PD controller-based method, the online learningbased method, the $\boldsymbol{F D A S H}$, the proposed framework with $\boldsymbol{I A M S}$, and the proposed framework with IMMS, by using two extensively used $Q o E$ models [43] and [45], as shown in TABLE III.
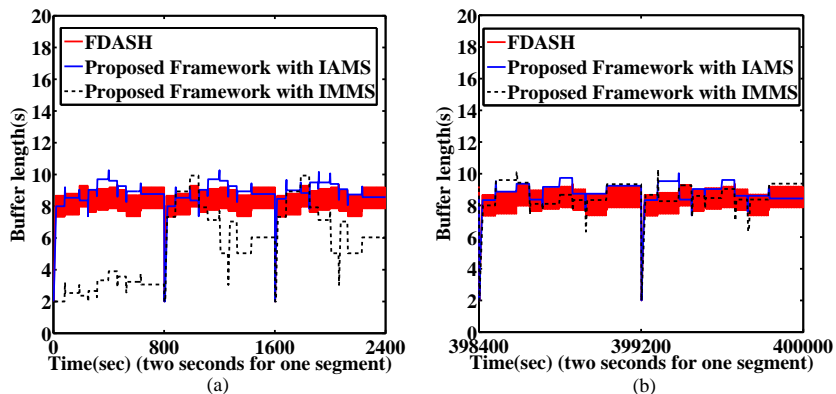

Fig. 29. Buffer length comparisons under the short-term fluctuating channel.
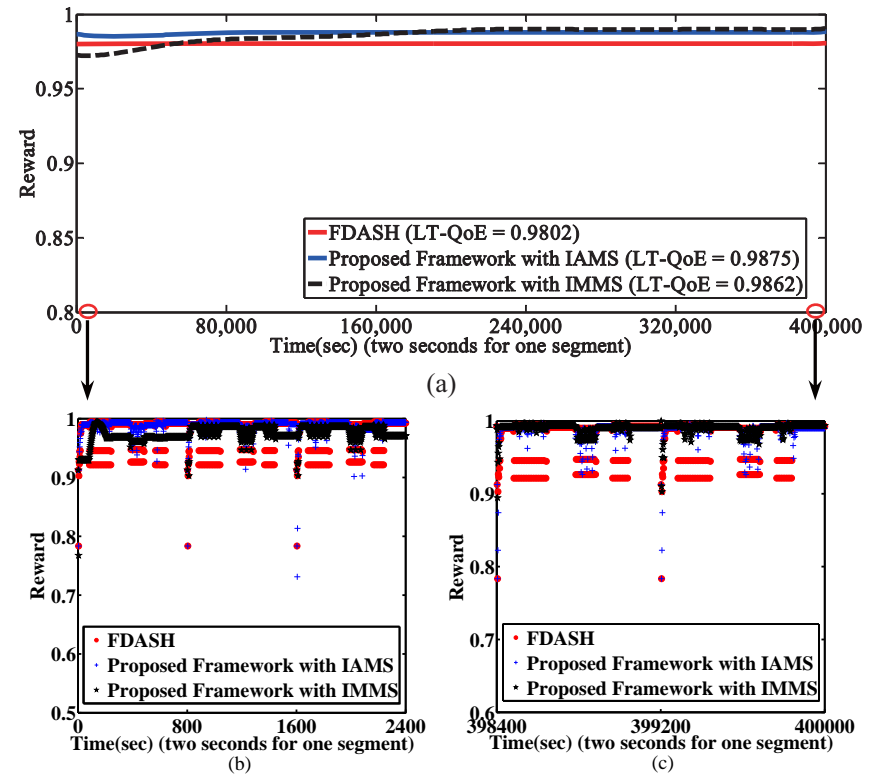

Fig. 30. Rewards and LT-QoEs comparisons under the long-term fluctuating channel.

The $Q o E$ model proposed in [43] can be written as

$$
\begin{array}{r}
Q o E^{M}=\sum_{m=1}^{M} q\left(r_{m}\right)-\lambda \sum_{m=1}^{M}\left|q\left(r_{m+1}\right)-q\left(r_{m}\right)\right| \\
-\mu \sum_{m=1}^{M}\left(T_{m}^{\text {down }}-B_{m}\right),
\end{array}
$$

where $\lambda=1$ and $\mu=6$ are model parameters that are empirically defined in [43], $M$ is the number of received segments, $r_{m}$ is the bitrate of the $m$-th requested video

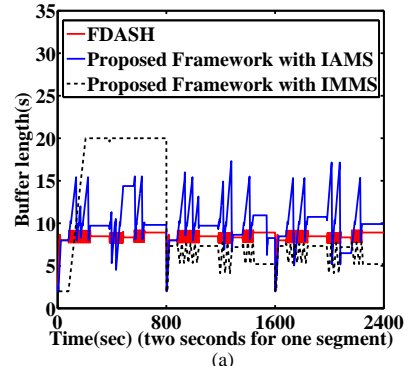

(a)

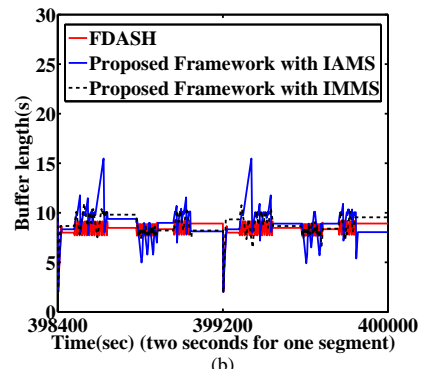

Fig. 31. Buffer length comparisons under the long-term fluctuating channel. 


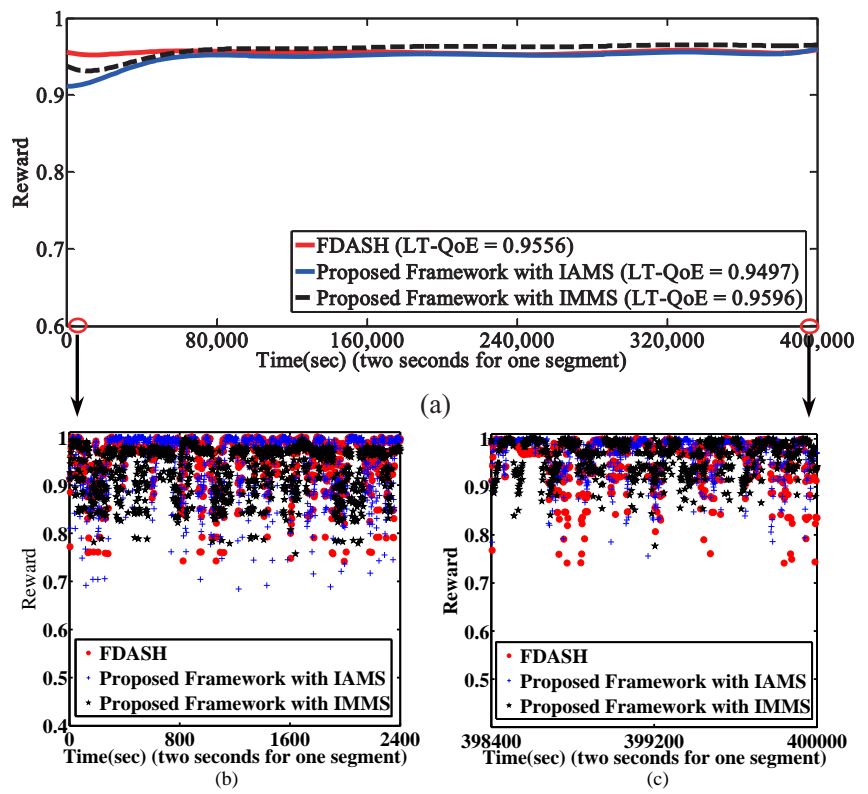

Fig. 32. Rewards and LT-QoEs comparisons under the Markov channel ( $p=$ $0.5)$.

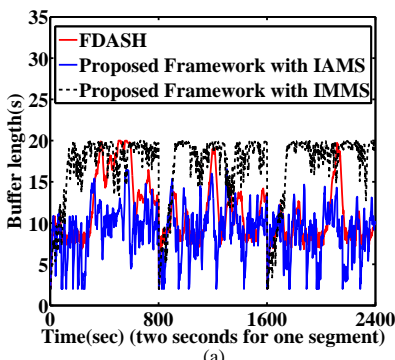

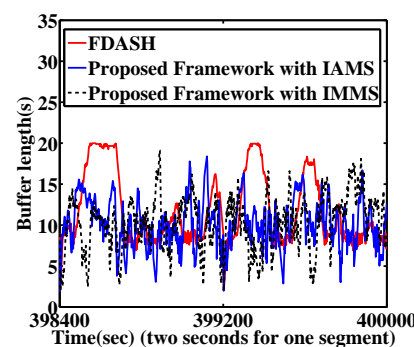

(b)
Fig. 33. Buffer length comparisons under the Markov channel $(p=0.5)$.

segment, $q\left(r_{m}\right)$ is the corresponding SSIM value, $T_{m}^{\text {down }}$ is the download time of the $m$-th segment, and $B_{m}$ is the buffer length at the end time of the $m$-th segment.

The $Q o E$ model proposed in [45] can be written as

$$
Q o E=4.85 \cdot Q_{n o r m}-4.95 \cdot F-1.57 \cdot S+0.5,
$$

where $Q_{n o r m}$ is the normalized average quality of all the received video segments,

$$
Q_{\text {norm }}=\frac{1}{M \cdot Q_{\max }} \cdot \sum_{m=1}^{M} Q_{m},
$$

$M$ is the number of requested video segments, $Q_{\max }$ is the highest quality of all the received video segments, $F$ is a factor to evaluate the negative gain of playout interruption,

$$
F=\frac{7}{8} \cdot \frac{\ln \left(f_{F}+1\right)}{6}+\frac{1}{8} \cdot \frac{\min \left(f_{T}, 15\right)}{15},
$$

$f_{F}$ and $f_{T}$ denote freeze frequency and average freeze duration respectively, $S$ in Eq. (14) is used to evaluate the negative gain of video quality fluctuations,

$$
S=\frac{s w_{\text {number }}}{M} \cdot \frac{s w_{\text {depth }}}{\left(Q_{\max }-Q_{\min }\right)},
$$

\begin{tabular}{|c|c|c|c|}
\hline Bandwidth Variations & Methods & QoE [43] & QoE [45] \\
\hline \multirow{6}{*}{$\begin{array}{l}\text { Constant channel } \\
\text { (3000kbps) }\end{array}$} & Rate based & 1.9705 & 4.5355 \\
\hline & PD controller-based & 1.9747 & 5.1679 \\
\hline & Online leaming & 1.9768 & 4.8388 \\
\hline & FDASH & 1.9730 & 4.8201 \\
\hline & Proposed Framework with IAMS & 1.9863 & 5.2042 \\
\hline & Proposed Framework with IMMS & 1.9862 & 5.0534 \\
\hline \multirow{6}{*}{$\begin{array}{l}\text { Short-term } \\
\text { fluctuating channel }\end{array}$} & Rate based & 1.9744 & 4.8001 \\
\hline & PD controller-based & 1.9797 & 4.9169 \\
\hline & Online leaming & 1.9764 & 4.8535 \\
\hline & FDASH & 1.9758 & 4.8307 \\
\hline & Proposed Framework with IAMS & 1.9819 & 5.0996 \\
\hline & Proposed Framework with IMMS & 1.9819 & 4.9944 \\
\hline \multirow{6}{*}{$\begin{array}{c}\text { Long-term } \\
\text { fluctuating channel }\end{array}$} & Rate based & 1.9740 & 4.4173 \\
\hline & PD controller-based & 1.9742 & 4.8526 \\
\hline & Online leaming & 1.9754 & 4.8037 \\
\hline & FDASH & 1.9749 & 4.7962 \\
\hline & Proposed Framework with IAMS & 1.9895 & 5.0621 \\
\hline & Proposed Framework with IMMS & 1.9894 & 4.9926 \\
\hline \multirow{6}{*}{$\begin{array}{l}\text { Markov channel } \\
\quad(p=0.5)\end{array}$} & Rate based & 1.9084 & 3.8498 \\
\hline & PD controller-based & 1.2075 & 2.2954 \\
\hline & Online leaming & 1.9290 & 3.9163 \\
\hline & FDASH & 1.9125 & 3.8658 \\
\hline & Proposed Framework with IAMS & 1.9353 & 3.9414 \\
\hline & Proposed Framework with IMMS & 1.9310 & 4.0930 \\
\hline \multirow{6}{*}{$\begin{array}{c}\text { Abrupt } \\
\text { channel changes }\end{array}$} & Rate based & 1.9451 & 4.2147 \\
\hline & PD controller-based & 1.6734 & 3.8982 \\
\hline & Online learning & 1.9546 & 4.4915 \\
\hline & FDASH & 1.9514 & 4.4706 \\
\hline & Proposed Framework with IAMS & 1.9533 & 4.4978 \\
\hline & Proposed Framework with IMMS & 1.9603 & 4.7388 \\
\hline \multirow{6}{*}{$\begin{array}{c}\text { Abrupt } \\
\text { video complexity } \\
\text { changes }\end{array}$} & Rate based & 1.9517 & 4.3494 \\
\hline & PD controller-based & 1.9539 & 4.5171 \\
\hline & Online leaming & 1.9583 & 4.5011 \\
\hline & FDASH & 1.9567 & 4.4792 \\
\hline & Proposed Framework with IAMS & 1.9691 & 4.5795 \\
\hline & Proposed Framework with IMMS & 1.9696 & 4.6614 \\
\hline \multirow{6}{*}{$\begin{array}{c}\text { Abrupt } \\
\text { channel and } \\
\text { video complexity } \\
\text { changes }\end{array}$} & Rate based & 1.9076 & 4.0211 \\
\hline & PD controller-based & 1.6498 & 3.8500 \\
\hline & Online leaming & 1.9160 & 4.0995 \\
\hline & FDASH & 1.9147 & 4.0830 \\
\hline & Proposed Framework with IAMS & 1.9245 & 4.3124 \\
\hline & Proposed Framework with IMMS & 1.9250 & 4.4776 \\
\hline
\end{tabular}

TABLE III

COMPARISONS RESULTS BY TWO EXTENSIVELY USED QoE METRICS

where $s w_{\text {number }}$ and $s w_{\text {depth }}$ represent the number and the average amplitude of bitrate switches, respectively.

From TABLE III, we can see that the proposed frameworks always produce the highest $Q o E s$ with respect to different types of channel bandwidth variations, no matter what $Q o E$ model is used. It can also be observed that for more complex bandwidth conditions and video contents, the performance of the proposed framework with $I M M S$ is better than that of the proposed framework with $\boldsymbol{I A M S}$.

\section{Conclusions And Future Work}

We proposed an ensemble rate adaptation framework for DASH clients by leveraging the advantages of multiple rate adaptation methods to improve the $Q o E$ of users. The proposed framework consists of two modules: a method pool and a method controller. At each method switching time, the method controller will select the best method that can achieve the best reward from the method pool. We also proposed two method switching strategies: IAMS and IMMS, to select an appropriate rate adaptation method. Three popular rate adaptation methods (rate-based method, PD controller-based method, and online learning-based method) are integrated into 
the proposed framework. Simulation results have shown that the proposed framework can fully use the advantages of the methods in the method pool, and always provide the largest $Q o E$ for users.

In the future, we will implement the algorithm in a more realistic scenario, based on a real test-bed which uses a realistic TCP transmission. The performance of the proposed ensemble learning-based rate adaptation framework largely depends on the integrated methods in the method pool. Therefore, we will add more advanced adaptation methods into the framework and improve the strategy of the method controller to adapt to more complex environments. Another future work is to cut down the system complexity and reduce the decision time as much as possible.

\section{ACKNOWLEDGMENT}

The authors would like to thank Institute of Information Technology (ITEC) at Klagenfurt University for the valuable and basis work of DASH.

\section{REFERENCES}

[1] T. Stockhammer, "Dynamic Adaptive Streaming over HTTP - Standards and Design Principles" in Proc. ACM Multimedia Systems Conference, California, Feb. 2011, pp.133-144.

[2] J. Kua, G. Armitage and P. Branch, "A Survey of Rate Adaptation Techniques for Dynamic Adaptive Streaming over HTTP" IEEE Commun. Surveys and Tutorials, vol. 19, no. 3, pp. 1842-1866, 2017.

[3] M. Dräxler and H. Karl, "Cross-Layer Scheduling for Multi-Quality Video Streaming in Cellular Wireless Networks," in Proc. the $9_{\text {th }}$ Int. Wirel. Commun. and Mobile Comput. (IWCMC), Sardinia, pp. 11811186, 2013.

[4] F. Dobrian, V. Sekar, A. Awan, I. Stoica, D. Joseph, A. Ganjam, J. Zhan and H. Zhang, "Understanding the impact of video quality on user engagement," in Proc. ACM SIGCOMM Conference(SIGCOMM'11), Toronto, pp. 362-373, Aug. 2011.

[5] H. Bai, W. Lin, M. Zhang, A. Wang and Y. Zhao, "Multiple Description Video Coding Based on Human Visual System Characteristics," IEEE Trans. Circuits Syst. Video Technol., vol. 24, no. 8, pp. 1390-1394, Aug. 2014.

[6] X. Yin, A. Jindal, V. Sekar and B. Sinopoli, "A Control Theoretic Approach for Dynamic Adaptive Video Streaming over HTTP," in Proc. ACM Conference on Special Interest Group on Data Communication (SIGCOMM), London, pp. 325-338, Aug. 2015.

[7] S. García, J. Cabrera and N. García, "Quality-Control Algorithm for Adaptive Streaming Services over Wireless Channels," IEEE J. Sel. Topics Signal Process., vol. 9, no. 1, pp. 50-59, Feb. 2015.

[8] D. Ghadiyaram, J. Pan and A. C. Bovik, "A Subjective and Objective Study of Stalling Events in Mobile Streaming Videos," IEEE Trans. Circuits Syst. Video Technol., vol. PP, no. 99, pp. 1-1, Nov. 2017.

[9] N. Eswara, K. Manasa, A. Kommineni, S. Chakraborty, H. P. Sethuram, K. Kuchi, A. Kumar and S. S. Channappayya, "A Continuous QoE Evaluation Framework for Video Streaming over HTTP," IEEE Trans. Circuits Syst. Video Technol., vol. PP, no. 99, pp. 1-1. Aug, 2017.

[10] D. Zegarra Rodríguez, R. Lopes Rosa, E. Costa Alfaia, J. Issy Abrahão and G. Bressan, "Video Quality Metric for Streaming Service Using DASH Standard," IEEE Trans. on Broadcast., vol. 62, no. 3, pp. 628639, Sept. 2016

[11] J. De Vriendt, D. De Vleeschauwer and D. C. Robinson, "QoE Model for Video Delivered over an LTE Network Using HTTP Adaptive Streaming," Bell Labs Technical Journal, vol. 18, no. 4, pp. 45-62, Mar. 2014.

[12] Y. Liu, S. Dey, F. Ulupinar, M. Luby and Y. Mao, "Deriving and Validating User Experience Model for DASH Video Streaming," IEEE Trans. Broadcast., vol. 61, no. 4, pp. 651-665, Dec. 2015.

[13] N. Carlsson, D. Eager, V. Krishnamoorthi and T. Polishchuk, "Optimized Adaptive Streaming of Multi-Video Stream Bundles," IEEE Trans. Multimedia, vol. 19, no. 7, pp. 1637-1653, Jul. 2017.
[14] D. Yun and K. Chung, "DASH-Based Multi-View Video Streaming System," IEEE Trans. Circuits Syst. Video Technol., vol. PP, no. 99, pp. 1-1, 2017.

[15] X. Ge, L. Pan, Q. Li, G. Mao and S. Tu, "Multipath Cooperative Communications Networks for Augmented and Virtual Reality Transmission," IEEE Trans. Multimedia, vol. 19, no. 10, pp. 2345-2358, Oct. 2017.

[16] J. Xiao, M. M. Hannuksela, T. Tillo and M. Gabbouj, "A Paradigm for Dynamic Adaptive Streaming over HTTP for Multi-view Video," in Proc. the 16th Pacific-Rim Conference on Multimedia, Gwangju, pp. 410-418, Sept. 2015.

[17] C. Federico, D. Stefano, T. Laura and F. Pascal. "Online Learning Adaptation Strategy for DASH Clients." in Proc. the $7^{\text {th }}$ ACM International Conference on Multimedia Systems (MMSys), Klagenfurt, pp.7788, May. 2016.

[18] C. Zhou, C. W. Lin, X. Zhang and Z. Guo, "A Control-Theoretic Approach to Rate Adaption for DASH over Multiple Content Distribution Servers," IEEE Trans. Circuits Syst. Video Technol., vol. 24, no. 4, pp. 681-694, Apr. 2014

[19] T.-Y. Huang, R. Johari, N. McKeown, M. Trunnell and M. Watson. "A Buffer-Based Approach to Rate Adaptation: Evidence from A Large Video Streaming Service," in Proc. 2014 ACM Conference on Special Interest Group on Data Communication (SIGCOMM), Chicago, pp. 187198, Aug. 2014

[20] T.-Y. Huang, R. Johari and N. McKeown, "Downtown Abbey without The Hiccups: Buffer-Based Rate Adaptation for HTTP Video Streaming," in Proc. the 5th ACM SIGCOMM Workshop on Future HumanCentric Multimedia Networking (FhMN), Hong Kong, pp. 9-11, Aug. 2013.

[21] S. Meng, J. Sun, Y. Duan and Z. Guo, "Adaptive Video Streaming with Optimized Bitstream Extraction and PID-Based Quality Control," IEEE Trans. Multimedia, vol. 18, no. 6, pp. 1124-1137, Jun. 2016.

[22] C. Zhou, C. W. Lin and Z. Guo, "mDASH: A Markov Decision-Based Rate Adaptation Approach for Dynamic HTTP Streaming," IEEE Trans. Multimedia, vol. 18, no. 4, pp. 738-751, Apr. 2016.

[23] A. Bokani, M. Hassan, S. Kanhere and X. Zhu, "Optimizing HTTPBased Adaptive Streaming in Vehicular Environment Using Markov Decision Process," IEEE Trans. Multimedia, vol. 17, no. 12, pp. 22972309, Dec. 2015

[24] V. Martín, J. Cabrera and N. García, "Design, Optimization and Evaluation of a Q-learning HTTP Adaptive Streaming Client," IEEE Trans. Consum. Electron., vol. 62, no. 4, pp. 380-388, Nov. 2016.

[25] M. Claeys, S. Latré, J. Famaey, T. Wu, W. V. Leekwijck and F. D. Turck, "Design and Optimization of a FAQ-Learning-Based HTTP Adaptive Streaming Client." Connection Science, vol.26, no. 1, pp. 25-43, Jan. 2014.

[26] S. Petrangeli, M. Claeys, S. Latré, J. Famaey and F. De Turck, "A Multi-Agent Q-Learning-Based Framework for Achieving Fairness in HTTP Adaptive Streaming," in Proc. IEEE/IFIP Network Operations and Management Symposium: Management in a Software Defined World (NOMS), Krakow, pp. 1-9, May. 2014.

[27] J. Jiang, V. Sekar and H. Zhang. "Improving Fairness, Efficiency and Stability in HTTP-Based Adaptive Video Streaming with FESTIVE," in Proc. the 8th ACM International Conference on Emerging Networking Experiments and Technologies (CoNEXT '12), New York, pp. 97-108, Dec. 2012.

[28] Z. Li, X. Zhu, J. Gahm, R. Pan, H. Hu, A. C. Begen and D. Oran, "Probe and Adapt: Rate Adaptation for HTTP Video Streaming at Scale," IEEE J. Sel. Areas Commun., vol. 32, no. 4, pp. 719-733, Apr. 2014.

[29] C. Wang, A. Rizk and M. Zink, "SQUAD: A Spectrum-Based Quality Adaptation for Dynamic Adaptive Streaming over HTTP," in Proc. the 7th ACM International Conference on Multimedia Systems (MMSys), Klagenfurt, pp. 1-12, May. 2016.

[30] A. Mansy, B. Ver Steeg and M. Ammar, "SABRE: A Client Based Technique for Mitigating the Buffer Bloat Effect of Adaptive Video Flows," in Proc. the 4th ACM International Conference on Multimedia Systems (MMSys), Oslo, pp. 214-225, Mar. 2013.

[31] H. Yuan, X. Wei, F. Yang, J. Xiao and S. Kwong, "Cooperative Bargaining Game-Based Multiuser Bandwidth Allocation for Dynamic Adaptive Streaming Over HTTP," IEEE Trans. Multimedia, vol. 20, no. 1, pp. 183-197, Jan. 2018.

[32] H. Yuan, H. Fu, J. Liu, J. Hou and S. Kwong, "Non-Cooperative Game Theory Based Rate Adaptation for Dynamic Video Streaming over HTTP," IEEE Trans. Mobile Comput., Early Access , 2018.

[33] C. Maxim, L. Steven, F. Jeroen, T. Wu, V. Werner and D. Filip. "Design of a Q-Learning Based Client Quality Selection Algorithm for HTTP 
Adaptive Video Streaming," in Proc. AAMAS Workshop on Adaptive and Learning Agents (ALA), Saint Paul, pp. 30-37, May. 2013.

[34] C. Liu, I. Bouazizi and M. Gabbouj, "Rate Adaptation for Adaptive HTTP Streaming," in Proc. 2nd Annual ACM Multimedia Systems Conference (MMSys'11), San Jose, pp. 169-174, Feb. 2011.

[35] Y. Sun, X. Yin, J. Jiang, V. Sekar, F. Lin, N. Wang, T. Liu and B. Sinopoli, "CS2P: Improving Video Bitrate Selection and Adaptation with Data-Driven Throughput Prediction," in Proc. ACM Conference on Special Interest Group on Data Communication (SIGCOMM), Florianopolis, pp. 272-285, Aug. 2016.

[36] L. Liu, C. Zhou, X. Zhang, Z. Guo and C. Li, "Probabilistic Chunk Scheduling Approach in Parallel Multiple-Server DASH," in Proc. 2014 IEEE Visual Communications and Image Processing Conference, Valletta, pp. 5-8, Dec. 2014.

[37] S. Zhang, B. Li and B. Li, "Presto: Towards Fair and Efficient HTTP Adaptive Streaming from Multiple Servers," in Proc. 2015 IEEE International Conference on Communications (ICC), London, pp. 6849-6854, Jun. 2015.

[38] A. Zambelli, "MS-SSTR: Microsoft Smooth Streaming Protocol Technical Report," Microsoft Corp., Redmond, WA, USA, Jun. 2010. [Online]. Available: http://www.iis.net/downloads/microsoft/smooth-streaming

[39] S. Akhshabi, A. C. Begen and C. Dovrolis, "An Experimental Evaluation of Rate-Adaptation Algorithms in Adaptive Streaming over HTTP," in Proc. ACM Multimedia Syst., pp. 169-174, Feb. 2011.

[40] Adobe HTTP Dynamic Streaming (HDS), Adobe, San Jose, CA, USA, 2016. [Online]. Available: https://www.adobe.com/devnet/hds.html

[41] Apple HTTP Live Streaming, Apple, Cupertino, CA, USA, 2016. [Online]. Available: https://developer.apple.com/streaming/

[42] C.G. Healey and J. T. Enns, "Attention and visual memory in visualization and computer graphics," IEEE Transactions on Visualization and Computer Graphics, vol. 18, no. 7, pp. 1170-1188, Jul. 2012.

[43] X. Yin, V. Sekar and B. Sinopoli. "Toward A Principled Framework to Design Dynamic Adaptive Streaming Algorithms over HTTP," in Proc. the 13th ACM SIGCOMM Workshop on Hot Topics in Networks (HotNets), Los Angeles, pp. 1-1, Oct. 2014.

[44] L. Yu, T. Tillo and J. Xiao, "QoE-Driven Dynamic Adaptive Video Streaming Strategy with Future Information," IEEE Trans. Broadcast., vol. 63, no. 3, pp. 523-534, Sept. 2017.

[45] R. Mok, E. Chan and R. Chang. "Measuring the Quality of Experience of HTTP Video Streaming," in Proc. the 12th IFIP/IEEE International Symposium on Integrated Network Management (IM), Dublin, pp. 485492, May. 2011.

[46] Z. Wang, A. C. Bovik, H. R. Sheikh and E. P. Simoncelli, "Image Quality Assessment: from Error Visibility to Structural Similarity," IEEE Trans. Image Process., vol. 13, no. 4, pp. 600-612, Apr. 2004.

[47] D. Suh, G. Park, H. Ko and S. Pack. "Mobility-Aware DASH for Cost-Optimal Mobile Multimedia Streaming Services," in Proc. 2015 IEEE Conference on Computer Communications Workshops (INFOCOM WKSHPS), Hong Kong, pp. 57-58, Apr. 2015.

[48] E. Altman, K. Avrachenkov, C. Barakat and P. Dube. "Performance Analysis of AIMD Mechanisms over A Multi-State Markovian Path," Computer Networks, vol.47, no.3, pp.307-326, Feb. 2005.

[49] D. J. Vergados, A. Michalas, A. Sgora, D. D. Vergados and P. Chatzimisios. "FDASH: A Fuzzy-Based MPEG/DASH Adaptation Algorithm," IEEE Systems Journal, vol. 10, no. 2, pp. 859-868, Dec. 2015. 